\title{
Twenty-One Arguments Against Propensity Analyses of Probability
}

\author{
Antony Eagle \\ Draft; please cite published version, Eagle (2004): \\ http://dx.doi.org/10.1023/B:ERKE.0000023408.61887.6a
}

\section{Analysing Probability}

By 1660, Arnauld and Nicole could already deploy a recognisable concept of probability: ${ }^{1}$

... in order to decide what we ought to do to obtain some good or avoid some harm, it is necessary to consider not only the good or harm in itself, but also the probability that it will or will not occur, and to view geometrically the proportion all these things have when taken together.

(Arnauld and Nicole, 1996: pp. 273-4)

We can discern in this passage several core features of the concept: (i) probability is a mathematical measure of the possibility of the occurrence of events (ii) it is intimately connected with rational decision making (iii) its assignment to an event is not dependent on the actual occurrence of that event. Call this the pre-theoretical conception of probability: it makes no claim about what instantiates or embodies the probability of some event, it simply states that there is a useful notion that has these features. With the rise of classical statistical mechanics, this pre-theoretical conception of probability was given a home in science. Some minor alterations were made, but it was recognisably a precisification of that same pre-theoretical concept that was being used. ${ }^{2}$ It was at this point that empiricist philosophical

\footnotetext{
${ }^{1}$ Hacking (1975).

${ }^{2}$ John Burgess correctly points out that these remarks, and this paper, concern only objective probability - what is sometimes, I think misleadingly, called chance. (I regard chance as an analysis of objective probability, not a neutral term for that species of probability.) Credences certainly obey the same mathematical constraints to count as probability functions, but I am not proposing to attack the straw figure of a propensity analysis of credence. (Note added after publication.)
} 
scruples against modality came to bear on the problem of the empirical content of probability, ${ }^{3}$ but it would be wrong to think that the only desideratum was to adequately explicate the scientific role of the concept. For the pre-theoretical concept placed a great many restrictions on the intuitions that governed the acceptability of the scientific use of the concept. The platitudes that connect probability with other pre-theoretical concepts restrict how much we can treat 'probability' in science as a technical term, to be defined as one might wish.

Rather, as Hájek (1997) has emphasised, we should treat probability as a target for philosophical analysis, responsive to both scientific and commonsense conceptual economies. We wish to find an analysis of probability that makes the scientific use an explication of the pre-scientific use; but this project should not be mistaken for the project of discovering a scientific concept of probability. The second task had been performed exactly when we identified scientific probabilities with normed additive measures over the event spaces of scientific theories. But to make this formal structure conceptually adequate we need to give an analysis of both the explicandum and the explicatum.

An analogy might help here: as standardly interpreted by the Kripke semantics, the $\ulcorner\square\urcorner$ of $\mathbf{S 5}$ is a precisification of the pre-theoretical concept of necessity. However, merely giving various conditions on the box that makes it behave in roughly similar ways to necessity does not yield an analysis. The role of possible worlds in the Kripke models itself cries out for philosophical attention, in just the same way as the original pre-theoretical concept of necessity did. For example, modal realism provides one answer to both pre-theoretical worries about necessity and about the precisified notion of necessity in S5. I take it that the relationship between a putative analysis of probability, pre-theoretical probability, and the Kolmogorovian measure theoretical formalisation of probability has much the same structure. ${ }^{4}$

Carnap (1962) has a long discussion of what he calls 'explication' of a pretheoretical concept in terms of a scientifically precise concept. He gives a number

\footnotetext{
${ }^{3}$ Indeed, it was only at this point that a problem with the metaphysics of probability was even recognised: all of a sudden in the mid to late nineteenth century, various kinds of empiricist frequentist or subjectivist/epistemicist accounts of probability were on offer whereas the notion of unanalysed chance was relatively unproblematic (and underspecified) before this time.

${ }^{4}$ Let us note in passing that as standardly used, 'interpretation' is a misnomer for the activity of understanding the concept of probability. For Kolmogorov gave us an interpretation in the logical sense: a sentence $\operatorname{Pr}(A)=p$ containing the one uninterpreted function symbol $\operatorname{Pr}(\cdot)$ is true just when in the model, Pr is assigned to some additive function $P$ whose domain is a Boolean $\sigma$-algebra and whose range is the $[0,1]$ interval, $A$ is assigned to some member $a$ of the $\sigma$-algebra, and ' $p$ ' denotes the value $P(a)$ in the model. We already have an interpretation in this sense. When I use 'interpretation' below, it will be in the sense of 'analysis', not in this logico-semantic sense.
} 


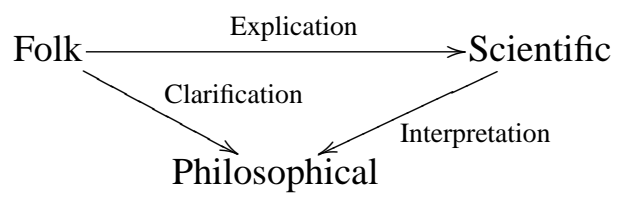

Figure 1: Relations between concepts in analysis.

of criteria: that the proposed explicatum (i) be sufficiently similar to the original concept to be recognisably an explication of it; (ii) be more exact or precise, and have clear criteria for application; (iii) play a unified and useful role in the scientific economy (so that it is not just gerrymandered and accidental); and (iv) be enmeshed in conceptual schemes simpler than any other putative explication that also meets criteria (i)-(iii). These are good constraints to keep in mind. However, this model is altogether too compressed: for it presumes that we have an independently good analysis of the scientifically precise concept (in effect, it suggests that scientific theories are not in need of conceptual clarification-that the "clear conditions of application' are sufficient for conceptual understanding). It also suggests that the explicatum replace or eliminate the explicandum; and that satisfying these constraints is enough to show that the initial concept has no further importance. But clearly the relation between the scientific and pre-scientific concepts is not so one-sided; after all, the folk are the ones who accept the scientific theories, and if the theory disagrees too much with their ordinary usage, it simply won't get accepted. I take this kind of approach to philosophical analysis to be pragmatist in some broad sense: it emphasises the conceptual needs of the users of scientific theories in understanding the aims and content of those theories.

The picture as I see it is that these four constraints operate to connect both the scientific and pre-theoretical concepts with their putative analysis, and it is through similarity of the analyses that we can identify the scientific concept as a precisification of the pre-theoretical concept. Both, however, stand in need of clarification or analysis however. I propose that the relation is something like that depicted in Fig. $1 .^{5}$

With an analysis in hand that satisfies Carnap's four constraints, we can then turn to external constraints on the analysis, not provided by the process of analysis or the concept under analysis. These will be constraints provided by our general philosophical outlook: whether the analysis is purely in terms of empir-

\footnotetext{
${ }^{5}$ Note that a single folk concept can have multiple analyses, due to the unavoidable vagueness of the folk concept.
} 
ically acceptable concepts, whether it gives reasonable criteria of application for the concept, and whether a concept like that deserves a place in our metaphysics. This is an important part of determining the adequacy of an analysis for a concept that plays a role in some larger conceptual economy. (To give an example: a nonHumean analysis of causation in terms of necessary connections between events might well satisfy all of Carnap's criteria, and yet be rejected for the "occultness" of its posited forces.) The overall aim will be to propose an analysis which best meets the internal and external criteria.

One note: when below I discuss the concept of probability, I mean by this no more than the relational property of probability (adopting a broadly Russellian semantics). I do not mean any kind of mental entity. I consider the task we are engaged in, namely the conceptual analysis of probability, to amount to the real definition of the property in terms of other properties that metaphysically constitute it (King, 1998). In this case, we have some folk property, which has some explication as a scientific property, both of which serve as constraints on what real metaphysical property can define and underlie facts about the unarticulated concepts. A correct metaphysical analysis will show that the property picked out by 'probability' is identical with the property picked out by the analysis. Sometimes there will not be a unique target for analysis, as in the case of probability with its many competing desiderata to be satisfied. In such a case we have a multiplicity of correct analyses, and which one is chosen depends at least in part on the rules for correct explication: just which concept we take the folk usage to be picking out. ${ }^{6}$

With these constraints on analysis in mind, let us turn to a family of putative analyses of probability ("propensity interpretations"). We shall see that, slippery creatures though they are, these analyses fail both internal and external kinds of tests for adequacy of a philosophical analysis, and must therefore be rejected.

\section{Propensity Analyses}

A frequency analysis of probability interprets statements about the probability of an event as a claim about the relative frequency of that event in some suitable collection of other events. Amongst philosophers of probability (though unfortunately not more widely), the problems with frequency interpretations of probability are well known and generally taken to be decisive. ${ }^{7}$ To avoid some of the perceived

\footnotetext{
${ }^{6}$ Thanks very much to Jeff Speaks for help with this.

${ }^{7}$ See Jeffrey (1977), Hájek (1997) and Hájek (unpublished) for many arguments against frequentism.
} 
failings of the frequency interpretation, Popper (1959b) introduced what he called the "propensity interpretation of probability". In particular, the propensity interpretation was to emphasise (i) how probability depended on the physical generating conditions of a sequence of outcomes; (ii) how probability could apply to the single case; and (iii) how probability is counterfactually robust. But satisfying these three desiderata is not enough to uniquely fix the content of propensity approaches. As with any substantive philosophical project, Popper's proposal soon splintered into many different subprojects. Popper's original paper actually contained hints of all the different forms that the interpretation would take, not clearly distinguished by him, so it still makes sense to talk of propensity interpretations as a family characterised by the motivating remarks he makes.

I shall begin with a general survey, paying particular attention to the three desiderata above; then, following Kyburg (1974), distinguish two primary variants of the propensity approach to probability: the long run propensity view and the single-case propensity view (which itself divides into two sub-variants). ${ }^{8}$ Those who feel familiar with the different kinds of propensity analyses should feel free to skip forward to $\S 3$, though I hope even the seasoned probabilist may find something of interest in the following discussion.

\subsection{From frequentism to propensity}

The shift from frequentism looks initially insignificant. Popper introduces the view as the inevitable consequence of a natural thing that the frequentist (at least, the frequentist who wants to interpret standard scientific practice) should like to say: that the admissible sequences of events (for the purposes of calculating relative frequencies) should be sequences of outcomes of repeated experiments, rather than arbitrary ordered collections of events.

Popper thought that propensities were necessary in quantum mechanics, contending that single case probabilities must be physically real relational properties of physical systems, that could interact with each other to alter the probability distributions over events directly, in order to explain interference effects and superpositions.

\footnotetext{
${ }^{8}$ See also Fetzer (1971). There are other, more idiosyncratic variants, such as the 'dispositional modal finite frequency' view of Jackson and Pargetter (1982), or the probability in branching spacetime view of Weiner and Belnap (2006). Some of the arguments below will apply to such views, insofar as they are propensity views at all; almost all of these idiosyncratic variants have other sui generis problems.
} 
However, Popper argues, we do not need to delve into the foundations of quantum mechanics to come across phenomena that require a propensity analysis, and he gives the following example. Take two dice, one biased $\frac{1}{4}$ towards sixes, the other fair. Consider a long sequence consisting almost entirely of throws of the biased die, interspersed with one or two throws of the fair die. What is the probability of a six on one of the throws of the fair die? According to frequentism, since the die tossing event is a member of a collective whose frequency of sixes is very close to $\frac{1}{4}$, the probability is $\frac{1}{4}$. But it is a fair die: so intuitively, the probability is $\frac{1}{6}$. This leads one to try and ensure that this mixed die tossing collective is not a collective: that some kind of objective homogeneity of the experimental apparatus is also required. This was already implicitly present in frequentist accounts of probability, but only as a pragmatic feature of the kinds of sequences that we would wish to accept for scientific purposes. Popper elevates this pragmatic methodological constraint into a metaphysical constituent of probability. (Note also that the frequentist cannot explain the plausibility of their methodological precept, whereas the propensity theorist can.)

This shifts probability from being primarily a relation between events and outcome sequences, to being primarily a relation between events and the 'generating conditions' underlying objectively homogenous sequences. These conditions are supposed to include the experimental apparatus, (perhaps some subset of) the ambient circumstances, perhaps the outcomes of previous trials; in any case, it is a property of some actual physical entities which is supposed to manifest itself in each single trial. It differs then from frequentism both in not relying on hypothetical entities like infinite sequences and being well-defined in the single case.

It is natural to take this property to be a dispositional property of the generating conditions, since the display of the characteristic features of a propensity is elicited only by subjecting the experimental apparatus to a certain kind of trial, rather than being always present. In what follows, I presuppose no particular account of dispositions. ${ }^{9}$ The categorical properties of the conditions may well underlie and ground the dispositional properties, but it is the disposition of the generating conditions to display a certain outcome just when the relevant kind of test is performed using the experimental apparatus that is supposed to ground probability

\footnotetext{
${ }^{9}$ Some accounts of dispositions will obviously be more amenable to some views of propensities. For instance, the 'dispositions as powers' view of Martin (1997), Shoemaker (1980), and Mumford (1998) might be more amenable to single case tendency analyses. Views like Prior et al. (1982), and Lewis (1997) in which dispositions supervene on categorical properties might be more compatible with long-run frequency views.
} 
assignments. ${ }^{10}$ This is what Peirce (1910) calls a 'would-be' of the dice, which he claims is exactly akin to a habit in an agent, and can provide the same explanatory resources. ${ }^{11}$ What the different propensity interpretations disagree on is what the firing of this disposition is, and how the disposition relates to the probability.

Note quickly how taking probability to be analysed in terms of a disposition enables it to meet the three Popperian desiderata of the opening paragraph of this section. To begin, it is a presupposition of the view that probability depends on the generating conditions of the event in question: such views trivially satisfy the first desiderata.

Secondly, if the probability derives from a disposition that is a continuing and stable property of the experimental apparatus, then it will be a property of the experimental apparatus even in the single case: even if the experiment is performed once and the apparatus is destroyed, the disposition will have still been activated and made its display. More carefully: the categorical properties that ground the disposition will have made exactly the same contribution to the single case event as they would have made in the long run. Perhaps the value of the probability cannot be ascertained by taking the single case as evidence; nevertheless the property of the experimental setup which constitutes the probability value would still have been wholly present.

Thirdly, since dispositions are supposed to be present even when they are not active (that's what makes them dispositional rather than categorical), they have a certain modal robustness that actual sequences do not have. We can say that even if the die is never thrown, were it to be thrown under standard conditions, then it would have a $\frac{1}{6}$ probability of coming up six, due to its propensity. There is no such modal claim to be made about actual sequences and what their frequencies would be, unless one has additional resources available to ground the modality. A dispositional propensity is one such resource.

How territorially ambitious are propensity theories? Objective physical probabilities at least are supposed to be grounded in propensities. Whether all other uses are also explained by propensities depends on the individual propensity theory in question.

\footnotetext{
${ }^{10}$ The die alone does not possess the property, but rather the entire die-thrower-surface system.

${ }^{11}$ Of course, there are further explanations that can only be provided when we look to the categorical properties that ground the dispositions, in the case of agents they might be psychological states, and in the case of dice, physical symmetries perhaps.
} 


\subsection{Long run Propensity}

The first variant is the long run propensity view. ${ }^{12}$ In slogan form:

Long run Events of type $A$ have probability $p$ iff the kind of experimental setup which can generate an $A$-event possesses a dispositional property to generate $A$-events with a characteristic relative frequency $p$ in the long run of trials of the setup.

In other words, were there to be a long run of trials of the experimental setup, it would be the case that the outcome sequence would have relative frequencies for each possible outcome that define the value of the propensity. This counterfactual is true in virtue of the possession by the experimental setup of some property governing the outcome sequence; it is dispositional because it is correctly ascribed through counterfactual claims. The possession of the property conveys to the experimental setup some power or capacity to generate outcome sequences with certain features.

Just how long is long? It seems fair to attribute to Popper and others the view that since the propensity is operative in every trial, we have an actual basis for describing hypothetical outcome sequences, and that we need not therefore be actualists as far as the long run goes as well. The propensity will be the non-modal ground of the assignment of a virtual frequency to the event-type in the long run sequences of trials of the apparatus. Therefore I take it that the long run is the infinite limit frequency:

$$
\operatorname{Pr}(A)=\lim _{n \rightarrow \infty}\left(\frac{\left|A_{s} \cap S_{n}\right|}{n}\right),
$$

where $\left\ulcorner S_{n}\right\urcorner$ denotes the first $n$ members of the outcome sequence $S,\left\ulcorner A_{s}\right\urcorner$ denotes the set of all $A$-events in the infinite sequence, and $\rceil \Gamma \Gamma$ denotes the cardinality of $\Gamma$.

It then needs to be the case that the virtual sequence of experiments forms a collective in the technical sense: an infinite sequence of outcomes with limit relative frequencies for each outcome (and often, but not always, the additional feature of randomness of the outcome sequence). ${ }^{13}$ Nevertheless, the frequencies in this view are seen as evidence for the existence of the propensity which produces them,

\footnotetext{
${ }^{12}$ Defended by, for example, Popper (1959b), Hacking (1965), Gillies (2000).

${ }^{13}$ Indeed, the view was largely present already in von Mises, who explicitly claimed that the empirical ground of the laws of collectives appeared in repeated experimental conditions.
} 
and not themselves the sole constituent of the probability — even if their values are exactly the same in virtue of the method of measurement adopted (infinite limits). The assignment of a propensity to a kind of trial guarantees that stable limit relative frequencies of outcomes will exist, produced by the interaction of the propensity and other properties of the trial apparatus.

Propensities are, according to Popper, properties of repeatable types of generating conditions - this is to ensure that the same propensity is realised at each particular token of an experimental setup. This is what enables us to assign a probability in the single case, despite the fact that the value of that probability is defined by modal facts about infinite long runs of trials. Finite trials and past experience are supposed to give us a sense of what the propensities are, which then allows us to infer to the system's behaviour in the infinite sequence of trials, and hence to the probabilities of types of outcome. The virtual sequence measures the value of the probability, but its ground lies in the propensity. The propensity doesn't itself possess a value: it is a sure-fire disposition to produce frequencies over the course of the long run of trials. Its role is to ensure that the relative frequencies so discovered will be correctly applicable to the trial in question, so the trial isn't of an inhomogeneous kind that should not correctly have a probability associated with it.

Popper takes it that the introduction of propensities as unobserved entities to explain the magnitude of frequencies is akin to the introduction of forces to explain the magnitude of observable events, like accelerations. Furthermore, just as in the case of forces, the introduction of propensities is supposed to be a empirical physical hypothesis: a propensity doesn't merely tease out the conceptual commitments of probability, but rather posits a particular physical instantiation of probability around here. Nevertheless, in any world in which there are probabilities, there will be some disposition which realises them-it is an analysis of probability since it provides a metaphysical real definition of probability in terms of other properties, even if those properties are picked out by description instead of rigidly. ${ }^{14}$

\footnotetext{
${ }^{14}$ In what sense is the claim substantive? If the claim is empirically substantive, then there are possible worlds in which it is false, hence there are worlds which it is not true of that the bearers of probability are dispositional properties. Such worlds would be worlds where the bearer of probability is something else. But if this is true, the claim that the propensity account is an analysis seems highly problematic. The analysis should yield a relational property sufficient to pick out probabilities in every world in which they occur, and be compatible with every way in which such probabilities might be realised (just as an analysis of pain should be compatible with every possible physical basis for pain). Perhaps then the claim is supposed to be metaphysically substantive. I take this to mean that it provides an adequate analysis of probability in every world where some probability ascription is true
} 


\subsection{Single Case Propensity}

The application of long run propensities to the single case remains a problem however. Even if we accept that the long run frequency has the value it has because of some propensity type, which is token realised in each trial, nevertheless the value of that probability always refers to the infinite sequence, without it being immediately clear what sense it would make to assign a probability value to a single trial. For instance, it would be an additional supposition that each trial is to receive the same probability value as the infinite sequence with the same propensity.

Popper speaks sometimes as if the dispositional properties which are token instantiated in each trial are in fact the real propensities, rather than token representatives of the long run propensity of the type of setup. This second thread was taken up, and the propensity was identified with whatever in the actual individual trial was active in bringing about the outcome. This single case propensity interpretation has two forms, which we distinguish. The first form, which we dub a tendency view of propensities, maintains that propensities are fundamental non-supervening properties that govern the production of probabilistic phenomena. The second form, due to Mellor, we dub the distribution display view; this view maintains that propensities supervene on other properties of the trial setup and are primarily proposed to explain the observed distribution of outcomes.

\subsection{Tendency}

Popper said:

[W]e do interpret probability measures, or weights attached to the possibility, as measuring its disposition, or tendency, or propensity to realise itself...

(Popper, 1959b: p. 36)

A natural interpretation of this remark is that the propensity is a kind of weakened or attenuated tendency for the generating conditions to cause or produce the outcome when trialed: ${ }^{15}$

of that world. But it must be noted that for this to be substantive, there must be some worlds where there are no probabilities. This commits one to somewhat controversial views about properties (that they do not exist in every possible world, or that relational properties can have contingent relations to monadic properties that they supervene on); it also commits one to the viability of fairly distant possible worlds (where partial belief isn't given a probabilistic analysis, there is perhaps only one event type, perhaps further odd claims). I rather think that the propensity theorist should give up on substantivity in this sense; analyses can still be controversial and epistemically substantive.

${ }^{15}$ See also Giere (1973), Giere (1976), and Popper (1990); a close variant is Fetzer (1981). 
Tendency An event $A$ has probability $p$ iff the actual token experimental setup which can generate $A$ possesses a dispositional property (tendency) to produce or cause $A$ to degree $p{ }^{16}$

The dispositional property is unlike many other dispositions, in that it does not always manifest when trialed. It is commonly thought that dispositional properties like fragility can be analysed into an ascription of a modal property of breaking whenever subjected to conditions $C$. But for this propensity, there will be no list of background conditions such that were the apparatus to be exposed to them, it would necessarily produce some outcome. No fixing of relevant causal factors will render the causal production sure-fire.

The analysis of this weakened causal production relation cannot itself be in terms of probability, lest the analysis be rendered circular. Frequency data has a role as evidence for the numerical magnitude of this tendency, with successive trials similar in the relevant respects (i.e. with all possible causally interfering factors held fixed) providing a firmer and firmer fix on the exact value of the propensity, as the evidence incrementally confirms some hypothesis about the strength of a causal relationship. But it remains possible that the frequencies, by chance, happen to confirm a false hypothesis about the magnitude of the casual relation.

Though the causal claim entails some claims about frequencies, it is not entailed by them. Its analysis must therefore consist at least in the provision of some other truth conditions. One may initially think that the notion of partial causation might help (in the sense that my fatigue and the glare were both partial causes of my car crash), but in fact this is not so. A partial cause is plausibly thought to be part of a complete explanation that would logically entail the event caused if given in its entirety. But even a complete probabilistic causal account will not entail that some particular outcome event occurred. And no account of partial causation has ever quantified the part-cause-of relation in the way that is required for probability.

There remain many possibilities: I will sketch a couple to give the general idea. (i) We could have a counterfactual analysis of the causal relation, and perhaps analyse the strength of the relation in terms of the proportion of nearby possible worlds with relevant features. $A$ causes $E$ to degree $p$ then obtains when amongst all the nearest $A$-worlds, $E$ occurs in $p$ proportion of them. ${ }^{17}$ (ii) Perhaps we have

\footnotetext{
${ }^{16}$ Compare: "The strength of the propensity of CSU [the chance setup] to produce outcome $E$ on trial $L$ is $r$ " (Giere, 1973: p. 471).

${ }^{17}$ This is a naive extension of Lewis (1973); the most sophisticated elaboration can be found in Pollock (1990). Pollock claims not quite to believe this view, but he does use it.
} 
a law of nature that entails a relation of 'probabilification' between states of affairs, or event types, or events of instantiation of universals, such that it is a metaphysical primitive that it has a degree equal to the probability of the outcome conditional on the trial. ${ }^{18}$ (Note both approaches normalise the degree of causation to the $[0,1]$ interval.)

If we take propensities to be causes in any of these ways, we shall have to admit a far wider class of relevant bearers of the propensity than the mere experimental apparatus. We know that causation is a complicated business, and that various factors can be causally relevant, either helping or hindering the production of some event, even when the primary cause is evident. ${ }^{19}$ So too, we should like to think that the die is the primary bearer of the property that constitutes the propensity, but that various other factors must collaborate in order for a trial to cause the outcome in question: the dice must be thrown correctly, gravity must be as it should be, and so on. Are these conditions all causes too, and do they get to be part of the experimental setup? Popper seems to favour taking the entire state of the universe to be the bearer of the propensity. Giere too abandons a quest that the long run propensity view perseveres with: to uncover exactly those features of a kind of trial which are statistically relevant for the outcome (i.e. attempting to partition the set of possible experimental apparatus into equivalence classes under homogeneity of frequency outcomes). Rather, the single case (perhaps an instantaneous state of the world) in all its particularity is the bearer of the propensity; this is putatively to avoid problems with classifying an event into a particular statistical class (see argument 9).

The view also yields a non-empiricist kind of causation; for we can consider two worlds identical in all particular events, and nevertheless assign to them different propensities. But perhaps this is not quite right; as I read Giere, he really wants propensities to be a new class of dispositional property that is particularly tied to causal phenomena; so two worlds that disagree on the value of propensities would disagree over some physical fact. It just turns out to be the case that this kind of physical fact is underdetermined by its effects, and doesn't supervene on other measurable properties. This makes propensities quite different from Newtonian forces, whose values are constrained by, and discoverable from, the measurable effects on acceleration of specified masses, or by measuring induced current, \&c.

${ }^{18}$ Perhaps along the lines of Armstrong (1997); for criticism, see van Fraassen (1989). Tooley (1987) uses logical probability (degree of entailment) between propositions about instantiation of universals.

${ }^{19}$ Field (2003) makes a related point. 
But the postulation of a physical property enables the neat integration of probability claims into broader scientific contexts. Giere can account for probabilistic independence in terms of causal isolation. He can account for methodological rules governing the choice of appropriate event space by pointing to the serious physical possibilities of the chance setup -in particular, highly unlikely possibilities can still be assigned probabilities despite the fact that in all likelihood they will not occur in the outcome sequence, hence wouldn't form part of the event space in a purely frequentist framework.

We should note too that this view emphasises the role of indeterminism in generating probabilities: for in a fully deterministic world, specifying the entire state at a time is enough to determine or fix completely the future evolution of the system; arguably, the only probabilities will be trivial 1 and 0 . Giere uses this as a stick to beat the frequentist, claiming that frequentists assign non-trivial probabilities even in fully deterministic cases, and hence must be subjective and rely on ignorance interpretations of probability. He himself advocates a fictionalist account of macrophysical probability in classical physics.

In any case, the account has to provide more argument to show how it actually functions as an interpretation of probability: for the physical magnitude of a propensity doesn't automatically satisfy a putative axiomatisation of probability unlike (some) relative frequency accounts (see argument 1). ${ }^{20}$

\subsection{Mellor's Distribution Display Account}

The other single case variant is a kind of hybrid view: it emphasises the role of the propensity in being completely fixed by the single case and the actual whole state of affairs, while taking over from the long run view the idea that a probability is given by a distribution over a partition of the event space, not the individual production of one event from that space. ${ }^{21}$

Distribution Display Event $A$ has probability $p$ iff the experimental setup which can generate $A$ possesses a dispositional property that warrants a subjective

\footnotetext{
${ }^{20}$ This is at odds with the contention of Kyburg (1974) that the dispositions in question 'fail to add anything to' the hypothetical limit frequency view. Kyburg mistakes the explanatory significance of the postulation of the propensity has for understanding the concept of a reference sequence and understanding frequencies: I therefore disagree with the semantical analysis of propensity statements he gives. I don't think that for Giere or the later Popper the 'almost-certainty' of a frequency claim exhausts the statistical content of a propensity ascription, since frequency claims are almost certain of 'pseudo-statistical' systems as well.

${ }^{21}$ This variant is defended by Mellor (1971), Mellor (1995).
} 
probability distribution Pr over a partition of outcomes including $A$, where $A$ has the value $p$ in the distribution (i.e. $p=\int_{A} \operatorname{Pr}(x) d x$ ) (Mellor, 1971: pp. 58-62)

Note that this disposition is sure-fire, producing some event that warrants some value of the distribution in every trial-avoiding some of the difficulties with the truth conditions for chancy dispositions. ${ }^{22}$ We should not think, just because a single event cannot reveal the full shape of the distribution, that the property which warrants that distribution is not fully displayed in each trial. That would be to make the frequentist mistake and identify the probability with the evidence.

Note also that the propensity is a disposition of the individual trial apparatus, not a disposition to produce outcomes distributed according to the distribution $\mathrm{Pr}$ over the long run of trials of the same type. The actual distribution over outcomes is evidence for the stable continuing underlying propensities of the objects involved in the trials. These stable propensities convey on their bearers very particular capacities to justify a probability distribution. Hence not just any constant physical arrangement that produces variable outcomes over time under repeated trials can have genuine propensities (unlike long run propensity views). In particular, Mellor's view is that genuine indeterminism is necessary for propensities.

Mellor proceeds somewhat differently from the other accounts we have discussed. He thinks that we begin by taking frequencies to constrain simple theories about the events in question, such that those theories can justify our rational expectations (our credences) in the events. He thinks that these theories will be constrained by the physical properties of the system in question, especially symmetries, through his principle of connectivity. ${ }^{23}$ Then through the Principal Principle (Lewis, 1980), we transfer this rational credence to the chance of the event in question, at which point we identify the propensity as just that physical property of the circumstances surrounding the event which makes our credence rational and undergirds the chance. One way to think about it might be to suppose that a propensity theory is the best explanation of our rational credences in cases where there is some objective ground to those credences (according to our best theories).

This kind of view can be contrasted with the single case tendency view by suggesting that the distribution displayed can supervene on the other physical proper-

\footnotetext{
${ }^{22}$ Thus, according to Mellor, the tendency views confuse one aspect of the display of the disposition (i.e. the production of some particular event of a partition) with the disposition itself. Note that there may also be some non-sure-fire disposition to produce a particular event; but that will not feature in the analysis of probability.

${ }^{23}$ Mellor (1971), p. 115. See Strevens (1998) for a related view.
} 
ties possessed by some system, in particular, the categorical properties discussed in the best theory of that system. The distribution is determined by some arrangement of occurrent categorical properties (i.e. symmetries) of the components of the system, and hence is manifested in each trial that subjects those properties to certain interactions. By contrast, tendency views postulate irreducible tendencies that do not supervene on any other occurrent properties of the system, but rather are dispositions inherent in the constituents.

Mellor (1995) develops this somewhat differently, taking the propensity to be that disposition of the chance setup picked out by Ramsifying the description "the property of the chance setup such that it governs the frequency $f_{A, n}$ of outcome $A$ in initial segment of the outcome sequence $S_{n}$ so that $\lim _{n \rightarrow \infty} f_{A, n}=$ chance(A)." This ensures that we identify that property which is probabilistically significant for the outcome sequence. This gives a constitutive connection between the outcome sequences, the chance and the propensity. This view moves closer to a 'theoretical term' view of probability, especially in the emphasis on the Ramsified description, and perhaps avoids some of the problems to be raised with his earlier account. ${ }^{24}$

Again, some similarities with the preceding views should be noted. Mellor thinks that an adequate theory of a deterministic universe would have us set the credences to either 1 or 0 if we knew all the relevant evidence; the specification of the complete state of the system means that the propensities are only trivial in deterministic worlds. And again, the postulation of properties that satisfy certain desiderata means that the analysis has empirical content and could be refuted by the non-existence of a class of properties with the features he demands.

\section{The Arguments}

Let us now turn to the arguments. Why so many? A philosophical analysis has to meet typically indistinct criteria for success, so no argument against any particular (consistent) analysis can be logically decisive. ${ }^{25}$ At best, one can make features of a proposed account explicit that are difficult to reconcile either with the internal constraints on the analysis or with other uses of the concept elsewhere. In this case, the number of distinct arguments indicates that there are a number of features of propensity analyses that have one of these problems. I think this will make clear the

\footnotetext{
${ }^{24}$ See argument 17.

${ }^{25}$ Even a good argument is resistible, as Armstrong is said to have remarked, but the more arguments of even dubious quality, the harder the resistance is to mount.
} 
potential cost of adopting a propensity analysis: that one has to make considerable adjustments to the concept one tries to explicate, and the resulting concept fits poorly into pre-existing roles for the concept of probability. Adopting a propensity analysis is thus a difficult and unappealing proposition, and no casual browser of potential interpretations of probability should adopt this one. At the least, I will have left the propensity theorist with a considerable burden to establish a tenable propensity interpretation within this framework.

I proceed as follows. I begin (\$3.1) with arguments that I take to weigh against all the analyses I canvassed in $\S 2$. I turn then to arguments that bear only on specific analyses: long run analyses in $\$ 3.2$, tendency analyses in $\S 3.3$, and Mellor's account in $\$ 3.4$.

\subsection{Against Propensity Analyses in General}

This section contains arguments designed to show that the concept of a propensity is a poor candidate to be used in an adequate explication of the concept of probability. This is both because the work propensities are required to do makes them a particularly problematic kind of property, and because their introduction into the analysis is mysterious. Note that there are no arguments against dispositions in general here. If such arguments in favour of the existence of only categorical properties were sound, then propensity interpretations wouldn't need 21 arguments against them, as they would be non-starters. I take it that dispositions are perfectly legitimate in many cases; I just think that propensities are not.

1. Establishing the axioms. There is a trivial requirement that any physical property that putatively provides a metaphysical correlate to probability assignments be interpretable as a mathematical probability. Typically, this means that the property should satisfy some standard axiomatisation of probability that supports the features of probability required by scientific practice.

All propensity theorists have been at pains to emphasise that what they are advocating is nothing less than a new category of physical disposition, introduced explicitly to play the correct metaphysical role in understanding science. Christopher Hitchcock suggested that there is a significant task for the propensity theorist to explain why some axiomatisation of probability holds of this new physical property.

Consider the pure subjectivist about probability. They have a certain claim about how their preferred interpretation of probability deserves the name: namely 
that degrees of belief must obey the probability calculus on pain of irrationality. They argue for this claim on the grounds that were one's degrees of belief not internally constrained by the probability calculus, then one would be vulnerable to a 'dutch book': a set of bets on hypotheses, each of which is individually fair by your lights, but that nevertheless leads one to a foreseeable sure loss. ${ }^{26}$

But it is completely unclear what could play the dutch book role for the propensity theorist in justifying the axioms. The propensities either need to primitively satisfy the axioms, or produce empirically accessible phenomena that do, like frequencies or credences. Neither option looks attractive, as I now show.

If the propensities are simply to satisfy some axiomatisation of probability in some brute way, then some serious empirical work would be required to establish that. But what guarantee is there that the reason propensities satisfy the axioms is a metaphysical or constitutive fact? In other words, what makes propensities an analysis of probability, rather than simply the empirical bearer of the concept of probability in this world?

If propensities are to satisfy the axioms in virtue of some other feature, then it needs to be shown that they possess this feature: that, for example, they can produce outcome frequencies of a kind that would support probability assignments. Again, this will involve investigation into the physical bearers of propensity.

In sum, by adverting to physical properties of systems in order to explain their probabilistic behaviour, propensity theories satisfy the intuition that probability is in some way connected to the objective situation. In doing so, they fall prey to an additional explanatory burden, namely giving a physical explanation for the obtaining of the mathematical facts about probability. It is difficult to see how they can satisfy this burden while remaining true to the idea that they give an analysis of probability, rather than a pseudo-scientific account of the particular facts that make probability ascriptions true of this-worldly events.

2. Disunity. Propensity theorists have emphasised that the introduction of propensities as a new physical category takes quantum indeterminism far more seriously than frequentists have. If propensities are the kind of thing possessed paradigmatically by quantum mechanical systems, then we know they must be properties quite unlike those we are familiar with, e.g. angular momentum or velocity. But we also know that angular momentum and velocity are significant among the properties on which the chances in coin tossing cases supervene. The question immediately

\footnotetext{
${ }^{26}$ Ramsey (1990), de Finetti (1964), Jeffrey (2004).
} 
arises: what kind of property is it, in virtue of which we deploy the concept of probability in both of these situations? For they are very different physical situations that both happen to have probabilities attached to them. So probability is a physically disunified concept, and the question should rightly be pressed as to whether propensity (as a putative physical realiser of probability) is disunified in the same way. If it is, then the operation of the property cannot be as straightforward as any of the accounts we have considered describe: there are, at least, no straightforward arguments in the literature about how radically different physical properties are supposed to instantiate the axioms of probability, and surely this is a burden the propensity theorist must try to discharge.

Perhaps the concept of propensity can be 'metaphysically unified', while the physical realisers are disunified. ${ }^{27}$ For example, although fragile things are fragile for many different reasons, an analysis of fragility as the disposition to break when struck is not thereby mistaken. The problem with propensities, however, is not whether there can be multiple realisers of propensities, but whether all those realisers will have enough features in common to be bearers of probability. The fragility disposition only applies to similar kinds of objects at a similar level of description and explanation. Each instance of fragility will have a different underlying constitution, but similar patterns of explanation, and similar structural features, will unite them. The way that quantum experimental setups have events occur within them is quite different to the way we take classically describable systems to have events occur within them. It is this worry that there will not even be structural features in common between the classical and the quantum propensities that really motivates this disunity objection. Consider the case where in some possible world, classical physics is correct, and compare that to a different world that is correctly described by quantum theory. Both worlds have empirical phenomena to which probabilistic theories correctly attach. But are there properties in common between these worlds sufficient to give a propensity interpretation to the probabilities that appear in each of the respective probabilistic theories? It is not at all clear to me that this is so, especially given that the probabilistic theories give perfectly acceptable accounts of the phenomena in question despite their diverse bases.

The concern is whether giving a metaphysically loaded slant to the probability ascriptions of such theories actually aids in their interpretation. It is worth pointing out in this connection that subjectivist and frequentist accounts of probability, whatever their flaws, have no problem with conceptually unifying probability

\footnotetext{
${ }^{27}$ Mark Johnston suggested that all propensities may be determinates of one determinable.
} 
ascriptions to radically varying kinds of events, since the constitution of the events is irrelevant to the grounds on which these interpretations ascribe probabilities. ${ }^{28}$

3. Determinism and Propensity. Of course, one way of resisting the demand for an argument that propensity is really one physically unified concept is to reject the claim that propensities exist in physical situations outside of indeterministic situations. Giere (1973) makes this move when he claims that a non-factive analysis of probability attribution in macroscopic situations is the only correct approach. Against this kind of suggestion, there are two points. Firstly, this contravenes the methodological precept we discussed at the beginning, that probability analyses need to be responsive to commonsense intuition. To reject probability for coins, dice or roulette is to clash drastically enough with the pre-theoretical concept that we might think some other concept is really being elucidated (analogy: imagine if we took the lesson of the analysis of temperature as mean kinetic energy to be that, outside of ideal gases, there is no correct assignment of temperature).

Secondly, it is not just commonsense intuition that is at risk from this move. Classical statistical mechanics proposes non-trivial probabilities, and yet is underlaid by a purely deterministic theory. To deny that these probabilities are 'real' is simply to come into conflict with one of the starting points of any genuine inquiry into the nature of probability: that it should explain the empirical success of probabilistic theories like statistical mechanics. It is a heavy burden on the propensity theorist to explain why these 'pseudo-probabilities', given that they are the best fillers of the role available (as far as explanation and prediction go), should be denied the umbrella of probability.

Popper (1990) wants to resist this kind of conclusion: he in fact wants to say that macroscopic events are indeterministic. ${ }^{29}$ But this seems an ad hoc manoeuvre at best, one which is not borne out by any analysis of the dynamics of statistical mechanics. ${ }^{30}$ I think that the correct response is to follow Clark (2001): "It seems to me that the issue of determinism versus indeterminism really ought to be (is) irrelevant to an interpretation of probability theory." (p. 275) But this is cold comfort to the propensity theorist who takes one of the prime motivations for their theory to be that it is so tightly entwined with indeterminism.

It should be noted that moving to quantum statistical mechanics provides no help, because of the very different ways that the two kinds of probability are stand-

\footnotetext{
${ }^{28}$ As a reviewer suggested.

${ }^{29}$ See Miller (1996).

${ }^{30}$ See Earman (1986), Sklar (1993).
} 
ardly taken to enter into the theory. One dissenting voice to this orthodoxy is Albert (2000), who argues (at 148-62) that classical statistical mechanical probabilities are none other than quantum probabilities as given by GRW theory. Even so, it seems metaphysically possible that there might be a purely classical world which is accurately described by classical statistical mechanics; this would be a world where probabilities accommodate determinism, and hence our analysis of probability must accommodate the possibility of determinism regardless of the constitution of statistical mechanics in the actual world.

4. Generalised Probability Spaces. Quantum mechanics is a probabilistic theory; but its probabilities may not be those of the familiar classical world. ${ }^{31}$ In particular, the additivity axiom does not in general hold: for quantum mechanical observables, $\operatorname{Pr}(A \cup B) \neq \operatorname{Pr}(A)+\operatorname{Pr}(B)-\operatorname{Pr}(A \cap B)$. This doesn't necessarily demand a revision in classical probability theory, but it seems to for the most straightforward understanding of how to generate probabilities for quantum observables (as opposed to classical measurement observables).

Of course, as van Fraassen (1991) points out (\$5.1), we can reconstrue the events in question so that the quantum mechanical probabilities of the observables having various values are conditional on measurement, rather than unconditional, and that these probabilities will satisfy the classical probability calculus. This is a controversial position from the point of view of the interpretation of quantum mechanics (Hughes, 1989). Moreover, this approach seems to be in some tension with the postulation of propensities, since it relies crucially on denying that probabilities can be assigned independent of measurement, yet the possession of a propensity is presumably going to be a measurement-independent property of the physical system, and not a property that is borne by the system only during a measurement. Even if we do grant that the propensity theorist can give an acceptable explanation for why they should adopt this conditional interpretation of measurement probabilities, it remains true that non-classical probability spaces do have a role in quantum mechanics, for example in Gleason's theorem, ${ }^{32}$ and it remains an open question for the propensity theorist to explain these uses.

If propensity interpretations are so dependent on indeterminism, and if (as we currently think) genuine indeterminism only arises in quantum mechanics, then

\footnotetext{
${ }^{31}$ This is for perhaps two reasons: either because the underlying algebra on the event space is an orthomodular lattice algebra, and not a Boolean algebra; or because of the existence of noncommuting operators, there are sets of operators, each of which have individually well defined probabilities, but fail to have joint probabilities. For details, see Dickson (1998); Hughes (1989), ch. 8.

${ }^{32}$ Hughes (1989), ch. 8.
} 
propensity interpretations are committed to failing to give an interpretation of classical probability spaces. More cautiously, propensity interpretations have no way of giving an empirical interpretation to the additional structure that classical probability spaces have, because the only empirical resources they allow themselves are sufficient only to constrain probabilities to a general probability space. Furthermore, extant mainstream propensity interpretations have aimed to validate the standard Kolmogorovian axiomatisation; since this axiomatisation fails in their preferred cases, they need to show that propensities satisfy the more general constraints. ${ }^{33}$

A propensity theorist might draw an analogy with non-Euclidean geometries, suggesting that quantum mechanics shows us that the real calculus of probabilities is non-additive, and that additive probability spaces are special cases that arise locally at macroscopic levels. ${ }^{34}$ But this will not help the propensity theorist. Even if a Euclidean space is a mathematically acceptable kind of object, that does not mean that there is any physical space that is Euclidean. Similarly, though additive probability functions are legitimate special cases of generalised probability functions, that does not mean that there are any physical propensities which give rise to additive probability functions. In the absence of any genuinely 'additive propensities', the propensity theorist cannot give an interpretation of classical probability spaces. The objection is that there seems no way for the propensity theorist to explain how probability could have been genuinely applied to classical probability spaces if propensities correctly interpret probabilities, except by mistakenly taking some non-classical propensities to be classical.

5. Finkish Propensities. Dispositions are malleable things: they can be altered by altering physical aspects of the bearers of the disposition. What if the displays of the disposition are some of the things that can alter the categorical basis? Consider a glass that if struck immediately anneals: until it is struck, it is fragile, but once it is struck it is not. Call such a disposition a finkish disposition. ${ }^{35}$

Consider now a finkish propensity: surely a possibility, since we have little information about what propensities are, except that they are a certain subclass of dispositions (and there is nothing that leads us to suppose that this subclass happens

\footnotetext{
${ }^{33} \mathrm{~A}$ reviewer points out that some propensity theorists have opted for a non-Kolmogorovian probability theory (Fetzer, 1981: pp. 284-5), and hence avoid this worry as stated. Yet this move runs into similar problems: for non-Kolmogorovian propensity interpretations make understanding the almost universally accepted Kolmogorovian probability calculus difficult.

${ }^{34}$ The analogy was suggested by Mark Johnston.

${ }^{35}$ Martin (1994); see also Lewis (1997).
} 
to exclude the finks). Whenever a finkish propensity is trialed, the outcome fails to happen, although there is a positive propensity for the outcome event to occur. Or consider a finkish propensity to produce a set of events $A_{1} \ldots A_{n}$, one of which $\left(A_{i}\right)$ retards its own occurrence.

This is a special case of causal interference in the manifestation of a disposition, in which the interference happens to exactly cancel the display. We cannot in general assume that the lack of manifestation is symptomatic of the lack of a disposition to manifest. ${ }^{36}$

Are we then committed to saying that, in this case, the generating conditions are not disposed to produce the outcome event after all? If we are to avoid this, we must abandon a counterfactual account of propensities in terms of their potential displays. But arguably this counterfactual element, while perhaps dispensable in the case of ordinary dispositions, is essential when dealing with a disposition that is supposed to underlie probability. This is because probability is closely connected with possibility. A non-trivial probability for some event means that event is seriously possible; that there is some world where it occurs. But if finkish propensities are possible, then some non-trivial probabilities lose this connection with possibility, since there will be no world where it is possible for the propensity to manifest without the fink blocking it. By contrast, dispositions of other kinds do not have the conceptual connection with possibility that probabilistic propensities are supposed to have, and hence seem less susceptible to the problem that if finked they do not possibly manifest. ${ }^{37}$

One possible response is to consider whether the finkish preventer is lawfully associated with the disposition. ${ }^{38}$ If it is lawfully associated, then perhaps we have a more complex disposition, not a finker. If it is not lawfully associated, then the connection between the finker and the disposition is not as tight as needed for the problem: perhaps the finker could be controlled for while the disposition manifests. The problem with the first alternative is that it is empirically underdetermined. If a coin, biased in favour of heads, also had a finkish propensity to prevent the landing of heads, such that the frequency evidence was even, we would not postulate any kind of complicated metaphysical setup to account for this. Rather, we would

\footnotetext{
${ }^{36}$ Imagine a similar circumstance: we have a drug that causes side effect $D$, and we wish to prevent the side effect from occurring. We could eliminate the tendency for the drug to cause $D$; or we could add some $D$-preventer to the drug. These are different causal situations, and different dispositions are active. We should not collapse them into each other.

${ }^{37} \mathrm{~A}$ reviewer pressed me on this point.

${ }^{38}$ This response was proposed by Mark Johnston.
} 
attribute a garden-variety propensity to land heads underlying a probability of $\frac{1}{2}$. The second alternative fails because it is supposed to be a feature of this disposition that it produces its own preventer: circumstances of controlling for the preventer while allowing the disposition to display seem unable to arise, and the connection with possibility is lost.

It seems that the finkish propensity can have necessarily defective empirical consequences, ${ }^{39}$ since every attempt to manifest it yields the wrong evidence for its value. This is surely a problem for a putatively scientific metaphysics: not only are we told that propensities are a new class of physical property, but we now conclude that sometimes there is no accurate evidence of the existence or operation of this property. We are given no reason at all to accept this confusing and complicated metaphysical entity, and we have every reason to look for other simpler theories which are immune to the problems. This line of objection, that propensity is a superfluous metaphysical posit, will come up forcefully in the discussion of single case propensities below (\$3.3); it is not often noted that it can arise more generally.

6. Conditional Propensities. On a propensity account, conditional probabilities, $\operatorname{Pr}(A \mid B)$, must be construed as conditional propensities: if the system produces outcome $B$, then it has a propensity to produce $A$ with a certain frequency, or with a certain degree of tendency, or in a suitable conditional distribution. On reflection this is quite strange. What we require is a conditional disposition: a dispositional disposition. It doesn't seem that a dispositional disposition is anything other than a disposition simpliciter however, with somewhat stronger conditions to elicit display, i.e. the disposition to produce outcome $A$ when trialed in a trial that produces outcome $B . .^{40}$

Let us introduce for this concept the notation $\operatorname{Pr}_{B}(A)$, intended to mean the probability of $A$ in the probability space generated by $B$ (i.e. the events are a $\sigma$ algebra of subsets of $B$ ). This probability space, I suggest, gives the correct formalisation of the conditional disposition for $A$ given $B$. For propensity theorists, specifying the physical situation gives the probability space; so there should really

\footnotetext{
${ }^{39}$ In the sense that, whenever it is possessed by an experimental setup, that setup produces misleading empirical data.

${ }^{40}$ Since (on Lewis-Stalnaker semantics) $p \square \leftrightarrow(q \square \rightarrow r) \vdash(p \wedge q) \square \rightarrow r$ when $p$ doesn't rule out the possibility of $q$ (and we should expect in the case under discussion that $p$ and $q$ are always compossible), dispositional dispositions turn out to be complicated ordinary dispositions. For dispositions as causal powers and similar views, we have an actual complex physical situation which is the bearer of the disposition; a disposition in the context of the firing of another disposition will be actually indistinguishable from a disposition with a more complicated condition for manifestation.
} 
be no such thing as conditioning on an event in the event space, but rather the construction of a new probability space based around the newly uncovered physical situation.

If we then stick to our original analysis of conditional probabilities, we are forced to admit $\operatorname{Pr}(A \mid B)=\operatorname{Pr}_{B}(A)$. But these two quantities are not in general the same. For by the ratio rule,

$$
\operatorname{Pr}(A \mid B)=\frac{\operatorname{Pr}(A \cap B)}{\operatorname{Pr}(B)}
$$

a well defined conditional probability is the ratio of well defined unconditional probabilities. But for the probability space generated by $B, B$ will not have a nontrivial probability. In $\operatorname{Pr}_{B}$, of course, it has probability 1: but that cannot be its value in an arbitrary probability space. There is in general no well defined probability for $B$. An example: there is a great difference between $\operatorname{Pr}(A \mid B)$ and $\operatorname{Pr}_{B}(A)$ when $B$ is the event "a fair coin is tossed" and $A$ is the event of "heads lands uppermost". Obviously $\operatorname{Pr}_{B}(A)=\frac{1}{2}$; but it is unclear what value the conditional probability has, since it is unclear what propensity there is for a coin to be tossed. This nonequivalence of concepts that the dispositions make equivalent is a problem for accounts that ground probability in dispositions. The propensity theorist only has the resources to account for one kind of conditional dependence of probability; but there are two.

The propensity theorist could argue that this simply means the ratio analysis of conditional probabilities is incorrect. There are independent grounds for thinking this. ${ }^{41}$ It must be noted that this is a significant disagreement with standard probability theory (Kolmogorov, 1956), and historically propensity theories have not analysed conditional probability directly. Furthermore, the kind of conditional dependence that propensity theories can provide (i.e. $\operatorname{Pr}_{B}(A)$ ) sets them at odds with others who have rejected unconditional probabilities and replaced them by primitive conditional probabilities, using, for example, Popper functions. The latter group continue to think that the ratio rule is a useful constraint on conditional probability, but there doesn't seem to be a straightforward way for the propensity theorist to analyse the propensity that underlies a ratio having a certain value.

7. Mathematical Propensities. The strong law of large numbers states that, with probability 1 , the long run frequency of attribute $A_{i}$ will have a limit that equals the

\footnotetext{
${ }^{41}$ See Hájek (2003b).
} 
probability $p_{i}$ of $A_{i}$. Then we have a probability of a probability (treating the firstorder probabilities as random variables), which on the propensity analysis seems to commit us to a disposition of a disposition.

That is fine. But the strong law of large numbers is a mathematical fact. Hence any disposition involved in making it true is a mathematical disposition. Since mathematical facts are widely supposed to be necessary, a straightforward modal account of such dispositions cannot be correct, unless dispositions that are necessarily firing when had - otherwise known as categorical properties — can be countenanced in the analysis of propensities. A counterfactual account seems to reduce to triviality unless we countenance 'impossible worlds' or suchlike. A causal account cannot be correct either since mathematical facts are the wrong kind of thing to be a relata of the causal relation. And the methodology of Mellor's approach seems not to get a handle on this situation. Indeed, the whole idea of a mathematical experimental setup is quite puzzling. Propensity theorists are left with an open question as to how to understand probabilities of probabilities, and not much prospect of answering it without either drastically altering their theory of dispositions, or abandoning the plausible claim that abstracta are causally inert. ${ }^{42}$

8. Quantification. Some people think that non-trivial probabilities apply to quantified sentences: 'The probability is 0.9 that all ravens are black'; 'the chance of there being a white raven is 0.02 '. But exactly what kind of propensity is there to make these sentences true? For the long run propensity, the problem is very difficult: as it stands, these sentences (e.g. 'all ravens are black') get made true all at once, eternally, so there is no sense to be made of repeated trials of them. So there cannot be a long run for these propensities to manifest.

Of course, perhaps such 'events' as all ravens being black do not have genuine probabilities, and the use of probability and chance in the sample sentences is purely epistemic. But consider if $90 \%$ of nomologically possible worlds would evolve (under the laws of nature) to situations where all ravens are black (given some natural measure over the possible initial conditions). That would seem to be evidence of the value of the conditional probability of all ravens being black given the laws of nature. It seems to be an objective probability assignment, and as such we would be correct to demand that propensity theories explain the basis for the correctness of that assignment. The propensity must be associated with the laws of

\footnotetext{
${ }^{42}$ Moreover, I don't think that retreating to the weak law of large numbers will help (as a reviewer suggested), since that law too discusses the mathematical behaviour of the probability of some mathematical fact involving another probability (Howson and Urbach, 1993: pp. 47-9).
} 
nature or with the initial conditions; but neither of these seem to be trialed multiple times.

In either case it seems we must have a single case interpretation of the relevant propensity. This too will face problems: what kind of thing is the 'generating condition' for this event? Perhaps it is the entire universe; or maybe just the initial conditions plus the laws of nature. Can such things be properly said to have a disposition to make it true that all ravens are black? It doesn't seem that an abstract entity like a body of laws, or a set of initial conditions, can have the relevant kinds of dispositions (see argument 7). Can there be a causal tendency analysis of the probabilistic truthmaking relation, as this seems to require? These questions remain wide open to the point where it is difficult to see any plausible account can be given that will resolve them.

\section{The Reference Class Problem. Reichenbach says:}

If we are asked to find the probability holding for an individual future event, we must first incorporate the case in a suitable reference class. An individual thing or event may be incorporated in many reference classes, from which different probabilities will result. This ambiguity has been called the problem of the reference class.

(Reichenbach, 1949: p. 374)

The problem is, given some particular event, which type of outcome should we classify it within to determine its probability? The reference class problem is typically taken to be a problem for frequentist analyses, where the 'type' of outcome determines the reference class and sequence to which the event belongs. Consider the event of a man's death in full particularity: his is presumably the only death to satisfy all and exactly these particulars. To get a probability, we need to generalise away from these particulars, to fix certain factors and vary others. For von Mises (1957) the single case chance of a man's death was 'meaningless'; for other frequentists, the single case chance was the chance of dying for a man qua smoker, the chance of dying for a man qua regular swimmer, $\& c$. The obvious problem is that competing reference classes yield different probabilities, with no reference class standing out as the 'correct' one. Not only does the event seem to have no determinate unconditional probability, but there is no guide for the rational agent to assign one based on evidence, despite many attempts to provide one.

Propensity theorists had hoped to avoid this problem by arguing that a complete specification of the physical situation, including all the propensities in question, would contain all the statistically relevant features of the situation, and would thus 
uniquely classify each single event into a probability space specified by the overall statistical import of the set of propensities.

There are in fact two problems with this, corresponding to each of the main propensity variants. Since they both aim to show that the reference class problem is a problem for propensities too, I include them here rather than in the more specific arguments. ${ }^{43}$ The dilemma will run as follows: if any event can be subsumed under more than one experimental setup or physical situation, as long-run propensity theories allow, then it will be possible that "qua event generated by such-and-such situation, $E$ has one propensity; qua event generated by another physical situation, it has a different propensity" (Hájek, 2003a: pp. 190-1). If an event cannot be subsumed under more than one experimental setup, as in single-case views, then there is relativity of propensity assignment to theoretical description, or else no way to generalise a result from one event to any other event, no matter how similar.

Long run propensity theories immediately inherit the reference class problem from the frequency analyses. The frequency interpretations have consistently failed to supply principles that would allow a unique reference class to be determined. For example, which properties would be relevant to the specification of the right reference class? Include too many properties, and our 'long run' of trials turns out to include just the single specific case we are interested in finding a probability for; include too few, and the number of reference classes which possess those properties and some combination of other properties multiplies the candidate frequencies. The consequent relativisation of probabilities to different sets of trials or sequences will immediately carry over to propensities whose values are fixed by those trials or sequences. Any candidate sequence which shares the relevant properties with the case we are interested in will have some propensity which produces its characteristic frequencies, and in virtue of being a member of each of these sequences, the case of interest will share the relevant propensities. ${ }^{44}$

\footnotetext{
${ }^{43}$ See Hájek (forthcoming) for arguments that every interpretation of probability faces a reference class problem.

${ }^{44}$ One hope remains for this proposal: that if propensities form a distinctive metaphysical kind, we could provide non-frequentist criteria for deciding which propensities were present in a given physical situation. This seems to be part of the idea behind specifying the generating conditions: the hope is that the probabilistically relevant features will thereby be fixed. However, this will not help. Firstly, there doesn't seem to be any means of detecting the presence of propensities apart from the probabilistic phenomena that the system enters into. Secondly, the epistemic problem about how to decide which propensities are relevant for the determination of the appropriate reference sequence remains unanswered. Blithely asserting the existence of unique 'generating conditions' won't help, because for long run propensity interpretations, every possible set of generating conditions will define a long run of some sort, with some defined probabilities for the events that occur in it.
} 
For single case propensity views, it is slightly trickier. As we have set up the positions above, every kind of propensity view takes the propensities to inhere in a set of generating conditions or in an experimental setup. Since the same kind of event can occur in more than one set of generating conditions, we have an immediate relativisation of propensity to a chance setup. Some propensity theorists might argue that this is alright, since every actual event will be produced by only one kind of generating setup, and that will fix the right reference class. This view is mistaken.

There are two kinds of single propensity theories, those which take the single case to have probabilities in virtue of non-propensity physical properties (like symmetries), and those which do not. For the first kind, typified by Mellor (1971), Hájek (forthcoming) argues that propensity will be inevitably relativised to a chance setup: the particular symmetries in question will end up determining the relevant probabilities. Consider symmetries as partitioning the outcome space; then different sets of symmetries provide different partitions, and combining probabilities from different partitions can lead in familiar ways to contradiction. For the chance set up of a repeated coin toss with outcome set $\{H H, H T, T H, T T\}$, one theory of the system will give propensity $\frac{1}{4}$ to each. But a different theory might take it that the relevant symmetries include the half-turn rotational symmetry, and count whether the nose on the head points left $L$ or right $R$ as different outcomes, which then gives 4 possible outcomes $(\{L L, L R, R L, R R\})$ for the toss of two coins, with propensity $\frac{1}{4}$ each. But unless we are to get violations of the additivity axiom, it had better not be the case that $H H=L L \vee L R \vee R L \vee R R$-we should instead relativise propensity assignments to outcome partition. ${ }^{45}$ The propensity for an event will then be theory dependent, and the set of tosses we are actually concerned with can be correctly described by each of them, with different propensities for the same outcome when described differently. In any case, propensities are not brute features of a setup; rather, they are relative to the partition on possible outcomes that the theory which assigns the propensity value to the experimental setup introduces. ${ }^{46}$

\footnotetext{
${ }^{45}$ Yet another theory might claim that the symmetries are permutation invariant outcomes: and the outcome set is then $\{H H, H T, T T\}$, each with propensity $\frac{1}{3}$. Note that the relative insensitivity of single case propensities to frequency evidence helps keep this symmetry theory viable in the face of the data. Furthermore, note that no a priori indifference argument can rule it out, since such distributions (so-called Bose-Einstein statistics) occur in quantum mechanics in an ineliminable way (van Fraassen, 1991: pp. 376-8).

${ }^{46}$ Indeed, any counterexamples to the Principle of Indifference that can be resolved by appeal to theory can yield a counterexample to reference-class independence for symmetries. See van Fraassen (1989), p. 303.
} 
This is in addition to the fact that the event-types in question are relative to a set of generating conditions, and that one and the same event can be subsumed under more than one experimental setup.

For the non-symmetry based, non-supervening theories, like Giere's, which are irremediably single case, though there is a unique singleton reference class, a related objection arises. This is what we might call the generalisation failure objection (Howson, 1984). ${ }^{47}$ The usual reference class problem is taken to be a problem about how to assign an individual event to a reference class. However, if brute single case chances are taken as primitive, there is a converse problem: how should we classify the statistically relevant properties and gain information about other trials from this one? Howson points out that to generalise the single case probability to a class of similar events, one needs to abstract away from some of the particular detail, while holding fixed the statistically relevant properties of the trial. But the notion of holding fixed makes no sense in the single case. Everything is (trivially) held fixed; there is no generalisation. The single case view solves the reference class problem only by trivialising it: everything has its own unique reference class. No rule is even envisaged that dictates how to apply inevitably partial knowledge of similar cases to this one.

Hajek (forthcoming) takes the lesson of these failures to be that conditional probability, conditional on a reference class or set of background conditions, is the fundamental notion, and we can only talk of unconditional probability when context fixes a conditioning event. I think this is roughly correct; ${ }^{48}$ nevertheless, it must be noted that very few propensity theories as they currently stand are formulated as based on axiomatisations of conditional probability. ${ }^{49}$ Indeed, part of the very motivation for many propensity theories was the idea that they could give us the unconditional probabilities that frequencies could not provide (i.e. independent of reference sequence). If they cannot, then one advantage over frequentism is lost.

\subsection{Against Long run Analyses}

10. Frequentism Revisited. The long run propensity view is closely tied to frequency analyses. As we shall see, this will be its downfall. Part of the problem

\footnotetext{
${ }^{47}$ See also Howson and Urbach (1993), p. 346.

${ }^{48}$ I have some reservations concerning whether we ever get to discharge the conditioning event in order to assign a direct unconditional probability, as it seems we must in some cases-for example, when deliberating.

${ }^{49}$ Popper (1959b) is one exception; see also his axiomatisation of conditional probability (Popper, 1959a: Appendix *iv).
} 
for frequencies is avoided because of the introduction of the generating conditions to undergird modal claims about probability. But frequencies fail for reasons other than their lack of counterfactual invariance, and these other reasons will carry straight over. ${ }^{50}$

We have interpreted the long run view as committed to infinite virtual sequences of outcomes within which to calculate the probability of an event. There are several problems with this view: ${ }^{51}$ (i) Order matters for infinite sequences: the infinite sequence $0,1,0,1,0,1 \ldots$ can be re-ordered $0,0,1,0,0,1 \ldots$; the limit frequency of 1 changes from $\frac{1}{2}$ to $\frac{1}{3}$. It may be replied that the temporal performance of the experiments gives a preferred order; regardless of whether this is so, it seems that something extrinsic to the generating conditions, namely, when they are activated, determines the frequency, while the probability is determined by no such thing. One might also wonder what feature of the generating conditions it is that constrains the temporal order of the non-actual trials! (ii) Given that these views rely on independent and identically distributed trials (though see argument $\mathbf{1 1}$ below), many sequences are possible. For a coin toss, the sequence $H H H H H \ldots$. is possible; so is the sequence THTHHTHHH ...; so is the sequence HTHHTTHHHHTTTT ... (i.e. $2^{n}$ heads followed by $2^{n}$ tails), etc. All of these sequences are possible, but all give the wrong answer. The first two give $H$ a limit frequency of 1, even though $T$ occurs infinitely many times in the second case. The third gives no limit frequency at all; the frequency oscillates between $\frac{3}{4}$ and $\frac{1}{2}$ for heads, but never settles down to a stable value. Now we have admitted that the long run frequency might be any value; the physical properties of the generating conditions which underlie the propensity don't fix the (hypothetical, counterfactual) value of the propensity. (iii) Perhaps at this point the long run propensity theorist will point to the abnormality of these sequences, and instead want to use the law of large numbers to show the typicality of the sequences with the right frequency. Firstly, this appeal is blatantly circular: for it presupposes that we have an independent grasp on the probability that appears in the LLN. Secondly, as Eells (1983) points out, there doesn't seem to be any other non-circular constraint in the vicinity. Once we have admitted that the disposition is not to exceptionlessly produce sequences with the right limit frequency, we have left open why there should be any statistical regularity at all in the sequences that are produced by trials of the

\footnotetext{
${ }^{50}$ There are lots of sub-arguments in this argument, some of which are independently devastating to these views.

${ }^{51}$ See Hájek (unpublished).
} 
apparatus. (iv) Finally, the limit frequency view is mathematically inadequate as an interpretation of probability: limit relative frequencies violate countable additivity, nor are they always defined over a given field. ${ }^{52}$

So we might retreat to the position that probability is the relative frequency within a finite virtual sequence; perhaps even the actual sequence of events. This might appear more viable, but actually introduces at least three further complications. (i) How long a finite sequence? There seems no privileged place to stop in the absence of the constraint that the trials be actual. (ii) Certain probabilities of events are inaccessible: for instance, finite frequencies are restricted to rational probabilities. But quantum mechanical probabilities can be real valued. (iii) Rounding errors: let a fair coin be (virtually) tossed only an odd number of times during its existence. Then the probability of heads will not be, indeed cannot be, one half. But this must be false if the coin is fair. We can also generate spurious dependencies the same way. Call $A$ and $B$ dependent iff $\operatorname{Pr}(A) \neq \operatorname{Pr}(A \mid B)$. Then we can get dependence without causation, so-called 'spurious dependence': simply consider a virtual sequence of $10 \mathrm{~B}$ 's and $7 \mathrm{~A}$ 's. Since these numbers are relatively prime, it is impossible for the events to have non-trivial equal probabilities, hence they must be dependent; but we concluded this without regard to the content of $A$ and $B .{ }^{53}$

11. The Jeffrey Problem(s). With regard to hypothetical frequency interpretations of probability, Jeffrey (1977) makes the following point (at §1). Such interpretations are committed to the truth-evaluability (indeed, the truth) of some counterfactual claims about what the coin would have landed were it to be tossed: namely, that it would have landed about $\frac{1}{2}$ heads were it tossed infinitely many times. Set aside worries about this particular counterfactual: focus on the idea that there could even be true counterfactuals about chancy situations. For if we think the coin toss is at all chancy, then we should also think that there is no fact of the matter about what the coin would have come up. Both 'were it tossed, the coin might have landed tails' and 'were it tossed, the coin might have landed heads' are true; so the corresponding 'would' counterfactuals cannot be true.

A very similar problem arises for long run propensity views in virtue of their reliance on frequencies. These views are committed to propensities providing objective constraints on the space of possibilities: that there is some definite answer to what would have manifested if this coin were tossed an infinite number of times.

\footnotetext{
${ }^{52}$ See van Fraassen (1980), p. 184.

${ }^{53}$ Hájek (1997) provides further arguments against finite frequentism.
} 
But to give a definite answer as to how a chancy coin would behave is to misunderstand chance. If the propensity is supposed to give a foundation for such a definite answer about how the coin would behave, then the propensity cannot be a correct analysis of chance.

The long run propensity theorist could reply here that the standard Jeffrey problem about the truth of certain counterfactuals doesn't quite apply. For it is perfectly compatible with there being no answer about how this coin would land on the next toss that there is an answer about how it will behave in the long run. Our intuitions about many counterfactuals allow for global constraints even when it is completely unclear what the local results will be (e.g. if a close election were to be held again, we may not know which candidate would win, but we know it would have been someone on the ballot).

The previous argument (10) tried to show that in fact there will be no determinate global fact about the hypothetical sequence either. Even if we now grant that there is such a fact, there remains a tension, as I will now attempt to show. This tension is closely related to the Jeffrey problem-instead of being a problem with the truth of certain counterfactuals about particular outcomes, the tension concerns the falsity of certain claims of counterfactual independence between trials. Both worries derive fundamentally from the fact that any long run view makes the probability or propensity of a chancy outcome in a particular trial depend on what happens at distant trials. ${ }^{54}$

The long run frequency governs only the global sequence of trials. Genuine chance seems to be accommodated, since each particular outcome could have come about differently. But there is a global constraint which means that despite appearances, these outcomes couldn't have varied too much: they can at best permute the outcomes so as to leave the frequency unchanged. If we consider some finite sequence $S$, and alter the first $n$ members to all 1's, a constraint is placed over the remaining members to adjust for the difference in frequency forced by that alteration. For an infinite sequence, the limit we take depends on the structure of finite fragments of the sequence; if part of some finite fragment distorts the long run frequency, the other parts must make up for it, in order that the taking of a limit will yield the correct long run value. In fact, then, the aberrant parts of a sequence impose a constraint over the other parts. If we consider that long run propensities are individuated by statistical properties of their displays, and if we consider them to be physical properties, then this very physical system could not have had a relative

\footnotetext{
${ }^{54}$ See the discussion of undermining in argument $\mathbf{1 3}$.
} 
frequency other than the one it did have, leaving the constraint very tight indeed.

This seems to indicate that first, the trials are not counterfactually independent, and second, the trials are not chancy. ${ }^{55}$ But these are basic constraints that need to be satisfied if we are to have an interpretation of physical probability.

12. Long run dispositions. There is a worry about what exactly it means for a disposition to be active in the long run of trials, but not through being active at every instance of the trials (remember, this is not a single case view). There is no problem with having different trials instantiate the same state of affairs and have the same propensities: the problem arises when ascribing physically potent dispositions to any abstract entities like kind of trials. How exactly does the propensity inherent in some state of affairs bring it about that the long run frequencies match the probabilities, without that propensity being simply constituted by the frequencies? Perhaps a kind of trial then cannot be an abstract object, but some temporally extended object, perhaps the fusion of all of the separate trials, so that the propensity can be causally active in 'forcing' the frequencies to limit to the correct values. But if the propensity is in fact not active in the single case, but only in the long run, the propensity cannot be identified with any local disposition of each trial. Either the propensity is itself a fusion of the dispositions of each trial; or the kind of trial must endure through time, with the propensity fully present at each moment. Both of these options seem controversial at best.

So maybe the disposition is like a law of nature, constraining the possible sequences by constraining the space of possibilities, rather than by causing the outcomes. But how a supposedly physical property could act so as to by itself constrain the space of possibilities is quite mysterious. And if it is a law of nature, then there are laws of nature for every distinct kind of experimental setup which evidences probabilities. But we see no such laws in our best physical theory, nor any more general laws from which such law could be derived.

\subsection{Against Single Case Tendency Analyses}

13. Non-Humean. Grant that our world has single case propensities that give rise to some of the observed features. Since single case propensities are not identified with the frequency evidence, there is a certain amount of flexibility as to what outcome sequence the propensity will produce. Then the values of the propensities

\footnotetext{
${ }^{55}$ Note that chancy here doesn't necessarily mean indeterministic. Even in a deterministic cointossing system, we should expect that counterfactuals as to what would happen if the coin were to be tossed are to be standardly evaluated in such a way that there is no determinate answer.
} 
could have been different and the observed frequencies could have been exactly the same. Therefore the observed frequencies do not fix the propensities. Single case propensity is non-Humean-it does not supervene on the arrangement of local observable matters of fact. ${ }^{56}$ I now show that while this is a particular problem for empiricist propensity theorists, it is also a problem for anyone who thinks probabilistic theories should be underminable, as it seems we should.

Reichenbach (1949) argues that because of this flexibility, the occurrence of any single event has no power to verify the assignment of any given probability to that event. Quite rightly this narrow verificationism has been rejected: the occurrence of a single event can incrementally confirm or disconfirm some probabilistic hypothesis. 57

But the empiricist spirit behind Reichenbach's argument remains appealing. Some contemporary metaphysical projects, in particular the project of Humean Supervenience of Lewis (1986), have retained this empiricism by requiring that local matters of fact about events at spacetime points ground every other fact. Frequencies do supervene in something like the right way; single case propensities do not. In virtue of this failing, they seem not to be able to effectively fulfil the other roles of probability. In particular, the use of probabilities to ground rational credences seems shaky unless the single case probability can connect with the expected run of events over time (see argument 14).

The single case propensity theorist might respond: since propensities are real properties, it is a matter of local occurrent fact whether one is present or not. This is not a problem for Humean Supervenience, since propensity forms part of the supervenience basis.

Now consider two distinct worlds that have exactly the same propensities for some events, and yet differ in the outcome sequences that occur. It seems we need to specify both the propensities and the actual events to specify the supervenience base of that world. But if we need to specify all the local occurrent events to specify the world, including the actual frequencies, then the further specification of propensities seems superfluous. It is difficult to see any further fact that doesn't supervene on the local occurrent matters of fact, except for the facts about propensities themselves. Propensities seem therefore to be introduced purely to supervene on themselves. Perhaps in the construction of a partial theory for the world

\footnotetext{
${ }^{56}$ Single case propensity also violates the constraint of Tooley (1987) that truthmakers for actual truths be themselves actual - the actual truth that this coin has a propensity relation of strength $\frac{1}{2}$ to produce heads will be true despite the failure of one of the relata to exist if the toss comes up tails.

${ }^{57}$ See Fetzer (1971).
} 
the postulation of propensities gives an explanatory advantage. But it is difficult at best to see what genuine metaphysical significance this kind of propensity can have in this kind of broadly empiricist metaphysical framework.

Many single case propensity theorists aren't metaphysical empiricists, so this argument won't faze them. But they must make some concession to empirical constraints in the realm of statistical hypothesis testing, and here it should be noted that they seem largely unable to do this. Following Lewis (1994), let us call a future history which is incompatible with the current assignment of chances to future events an undermining future. As Ismael (1996) has argued, underminability of a theory of chance is in fact a feature in its favour, since it shows a modicum of responsiveness to evidence. Single-case propensities are not underminable, and are thus radically insensitive to evidence. This is not the usual problem of characterising statistical inference, since in this case we have no connection whatsoever between the chances and the evidence. There are worlds where the frequency of $A$ is $p$, but for any $q, 0 \leqslant q \leqslant 1$, the chance of $A$ could be $q$. If this is so, then the problems of direct and inverse inference look completely intractable. ${ }^{58}$

14. Horizontal/Vertical Problem. A severe problem for the single case chance theory is that it fails to meet the minimal requirement that it guide rational expectation. ${ }^{59}$ The semantics for single case propensities in Giere (1976) involves a uniform distribution over the set of all alternative possible worlds whose history up until now matches our world. The first problem is how to justify a uniform equipossibility assumption for all possible worlds (rather than one based, for example, on a Lewisian similarity metric which would make closer worlds more probable). Set that technical issue aside.

The more significant problem is how this probability across different worlds at the same time is to apply to this world at different times: for example, how does it apply to future frequencies? Severing the constitutive link between frequencies and chances means that we have no logical connection between the concepts of probability and rational expectation. Since, as we have seen, the events that occur in a world and the chances of those events are not logically related, why should knowledge of the chances tell us anything about which events to expect to occur? There seems no way that these single case propensities can rationalise adherence

\footnotetext{
${ }^{58}$ See Eells (1983) for more on this theme.

${ }^{59}$ The canonical exposition is van Fraassen (1989); see also Clark (2001), Eells (1983), Salmon (1979), Lewis (1994), and Loewer (2004). It is raised by van Fraassen and Lewis as problems for the Armstrong/Tooley style account of nomic relations between universals, but applies to propensity interpretations more generally.
} 
to Lewis' Principal Principle or anything like it; but without the Principal Principle we have no link between the two major uses of probability. Lewis says: "Don't call any alleged feature of reality 'chance' unless you've already shown that you have something, knowledge of which could constrain rational credence" (Lewis, 1994: 239).

This problem essentially appeared earlier (argument 9). Consider each event in its full specificity. We already decided that there was a difficulty in generalising from the single case to any particular outcome sequence. One might dissolve this worry by just accepting that every event is sui generis. Then each particular event provides no constraint on any of the other events, not even which events are members of the relevant comparison class that its propensity gives information concerning. There is no constraint on rational expectation provided by the propensities, because there is no information about which events other than itself it can be taken to apply to. (The failure of single case propensities to generalise correctly is due to the fact that single case propensities aren't closely enough connected to the larger pattern of outcomes.) Simply, this shows that rational expectation and propensities can come apart in a way that rational expectation cannot come apart from probability—so propensities are not probabilities.

15. Humphreys' Paradox. This problem is devastating for views that take propensities to involve weakened or intermittent causation. This is because causation fails simple inversion theorems of the probability calculus. ${ }^{60}$

Consider Bayes' Theorem; let $\mathfrak{B}=\left\{B_{1}, \ldots, B_{n}\right\}$ be a partition of the outcome space, and $A$ some event. Then for each $1 \leqslant k \leqslant n$ :

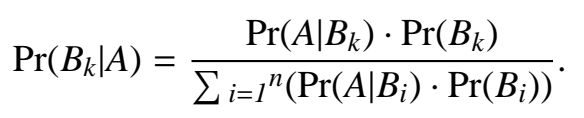

The most natural interpretation of conditional probability as a propensity is to consider the conditioning event as a type of experiment, and to consider the propensity of the conditioned event in that experiment. Humphreys considers an experiment with an electron source, a half-silvered mirror, and a receiver. There is an overall probability of electrons passing through the entire apparatus; there is a further probability of the electron hitting the receiver given that it passed the mirror, and this is most naturally construed as a conditional probability. But even if it made sense to consider the event of transmission through the mirror to have a propensity

\footnotetext{
${ }^{60}$ First pointed out in Humphreys (1985); see also Milne (1985).
} 
to bring about electron receiving events, the converse does not hold; the receiver does not have a propensity to bring it about that the electron was passed through the mirror. Or at least, the propensity should be 1 because an electron passing through the mirror must occur in order for the receiver to activate. But the inverse probability will not in general be 1 . So there is an asymmetry in propensities as causes that is not present in probability; so probabilities cannot be propensities.

The point is simple: the interpretation of probability should not require actual backwards causation for every well defined inverse probability!

Some attempts have been made to rescue propensities from the paradox. ${ }^{61}$ But these have mostly relied on weakening the causal conception of a tendency to a conception of propensities as tendencies for a system to produce outcomes. The response maintains that in Humphreys's argument, the propensity for the electron hitting the receiver given it passed through the mirror is a propensity before it passes through the mirror. Thus the propensity is for a system prepared in that initial state to have the events $S$ and $D$ co-occur, both at some future date, and both causally relevant to the current state.

This approach however will not help the single case tendency propensity theorist: for they want to interpret propensities as efficacious directly between physical states, and analyse 'efficacious' causally. Either they fail to interpret the probability calculus, ${ }^{62}$ or they weaken their position to some kind of 'chances of coproduction' interpretation of propensities. But this latter interpretation is subject to the problem of not providing much more than a redescription of the probability calculus, and the physical meaning of the interpretation is lacking.

At this point it is worth recalling argument $\mathbf{6}$, which claimed that the kind of conditional probability that propensity theorists are best able to capture is that of an event conditional on an event space. Humphrey's paradox seems to indicate that orthodox probability theory is committed to a kind of conditional probability which is not so closely connected to the physical realisation of the generating conditions. Christopher Hitchcock has pointed out that the propensity theorist really can't claim that their analysis of conditional probability is right, for their analysis doesn't seem to be able to explain how Bayes' theorem is true of probabilities-and Bayes' theorem is non-negotiable for the probability calculus. ${ }^{63}$

\footnotetext{
${ }^{61}$ See McCurdy (1996).

${ }^{62}$ Perhaps then adverting to a non-Kolmogorovian calculus, e.g.Fetzer (1981).

${ }^{63} \mathrm{~A}$ similar point applies to a proposal suggested to me by Hans Halvorson. He proposes that the propensity theorist should take some probabilities to be directly grounded in physical propensities, while others (such as the inverse probabilities in Humphreys' example) should be regarded
} 
16. Causal irrelevance and non-locality. An extension of the last problem. Let $A$ have a propensity to cause $B$, and let $C$ also have a propensity to cause $B$, but be causally isolated from $A$ - say, at spacelike separation, so that $A$ and $C$ are both in the past light cone of $B$, whereas $A$ appears in neither the past nor future light cone of $C$, and vice versa.

So $\operatorname{Pr}(B \mid A)$ and $\operatorname{Pr}(B \mid C)$ are both well defined; let us assume that the unconditional propensities are well defined also. Then, by (3), the inverse conditional propensities $\operatorname{Pr}(A \mid B)$ and $\operatorname{Pr}(A \mid B \wedge C)$ are well defined. Moreover, in general $\operatorname{Pr}(A \mid B \wedge$ $C) \neq \operatorname{Pr}(A \mid B)$, so $A$ is not probabilistically independent of $C$. So $C$ has some propensity significance for $A$, despite the fact that $C$ is causally isolated from $A$. So the tendency that propensities have to produce events cannot even be a causal tendency in this setting, unless the causation involves faster than light backwards causation; or causal influence from causally isolated events. Either way, this is difficult to accept.

17. The method of pure postulation. One way that the defender of tendency propensities could avoid many of the problems we have discussed so far is simply to stubbornly assert the existence of irreducible de re probabilistic dispositional properties as part of the fundamental furniture of the world. ${ }^{64}$ I have no knockdown argument against this robust sense of ontological entitlement (though see the next argument). But I think that the more methodologically modest of us would baulk at such a method. I will show that either propensity theorists are merely stipulating the existence of propensities, or else they are merely relabelling probabilities. Either way, they do not provide an analysis.

This method of stipulation has, in Russell's words, all the advantages of theft over honest toil. Unfortunately, as in all such cases, merely positing the existence of such a new category of physical property will fail to establish the existence or uniqueness of the properties in question. At the minimum any such claim should involve at least some empirical research. Admittedly, we have some evidence for

as mathematical constructs without direct correspondance to a propensity of their own. This twotiered model of propensities, while it may help avoid the problem of inverse tendency propensities, will produce significant difficulties for the propensity theorist in justifying the Kolmogorov axioms. If Halvorson's suggestion were adopted, only some objective probabilities would be grounded by propensities. Hence propensities determine a class of functions that strictly includes probability functions, and we need some other objective constraint to capture all and only the probability functions. If that constraint is not propensities, then what? And if something else can be found, can that something else possibly conflict with a propensity, or render the propensities dispensable? The problems multiply. (Note added after publication.)

${ }^{64}$ David Chalmers pressed this option on me. 
the existence of such a category, in the usefulness and applicability of probability itself. But such evidence is inconclusive at best, as the existence of other interpretations of probability shows. It is certainly not enough to establish on conceptual grounds alone the truth of any contingent existence claim about the kinds of properties which feature in the physical world.

One way to escape this charge is to suggest that nothing substantive has really been said. Consider some probabilistic theory, characterised by a class of probabilistic models. Some of the properties of the objects in the model, it will be claimed, are propensities. There must be some property that underlies the objectivity of assignments of probability; let that property be henceforth dubbed a propensity. This kind of response looks promising. ${ }^{65}$ Unfortunately, the propensity theorists we have looked at are not content with merely picking out some theoretical entity by a description, but have proceeded to give substantive analyses of that entity. These analyses have presupposed that 'propensity' picks out a non-gerrymandered class of properties, unified by their kinds of causal powers and by their relations to certain kinds of categorical properties of display events.

This however cannot be right. At best this shows that propensities can be an explication (in Carnap's sense) of the pre-theoretical notion. The identification of these features of probabilistic models with the concept of propensity and hence the construction of a propensity analysis is a further task. That this is so can be seen by looking at frequentist analyses of the very same probabilistic models: they replace the probabilistic properties of objects with non-dispositional properties of a different kind of object altogether. So this strategy cannot ground a propensity analysis.

Just as dubbing whatever property that some drug has to put one to sleep, 'dormitive virtue', fails to tell us anything new about that property, so dubbing some physical property 'propensity' gives us no grounds for an substantive claim whatever about that property. (Given the existence of grue-like predicates, even the claim that 'is a propensity' picks out some unified class of properties is debatable.)

Suppose that frequentism is true. Then there is a property of a class of events that underlies ascriptions of objective chance: the property " $x$ forms a collective which is objectively random". Then frequentism is a kind of propensity analysis! Or rather, a 'propensity analysis' in this sense is just an objective analysis of probability, nothing more.

\footnotetext{
${ }^{65}$ Indeed, some of those influenced by Mellor's use of Ramsifying have taken chance to be defined as a theoretical term in just this way: see Lewis (1980), Levi (1980), and Levi (1990).
} 
Because it bears this trivial relationship to probability, no proposed feature of propensity can explain any of the features of probability. The attribution of any particular features to a propensity is illegitimate if it is introduced in this purely non-constructive way. The fact that probability has a pre-existing content doesn't help to pin down propensities either, since part of the task of analysis is deciding how much of the pre-existing concept can survive in a philosophically rigorous framework. The semantic content of the description to be Ramsified remains just as unclear as the pre-theoretical concept, and is no advance over it. ${ }^{66}$ This kind of 'propensity analysis' is itself at best a placeholder for a fully spelled out analysis that the propensity theorists have as yet failed to give us.

In sum, the method of pure postulation either ends up in the position where substantive facts about probability are simply claimed to hold without argument, or no analysis has been given. One is methodologically unsound, and the other doesn't begin to address the question of analysing probability with which we started. ${ }^{67}$

18. $\boldsymbol{D e} \boldsymbol{R} \boldsymbol{e}$ ? If propensities are de re probabilistic properties, then their bearers must be res: i.e. objects. But there are sometimes too few, and always too many, objects of a propensity ascription.

To begin, propensities are properties of an experimental setup. A sensible thought about a complex experimental apparatus is that its properties should supervene on the properties of its parts. So the propensity of the whole apparatus must supervene on the properties of the parts, so the propensity won't be a simple irreducible property after all. If we deny the supervenience thesis, and argue that probability is an intrinsic emergent property, then the bearers of this primitive de re property aren't at all like the entities we usually ascribe such properties to. This is because an enormous set of objects (facilitators, possible confounders, \&c.) combine to constitute the precise ground for the production of the outcome. Each object in this set is a bearer of the propensity, but not in virtue of each object's making some partial contribution. A property of a complex that nevertheless doesn't su-

\footnotetext{
${ }^{66}$ Recall the first section: philosophical analysis is not merely a matter of mechanically deconstructing the concept.

${ }^{67}$ Thanks to Daniel Nolan for clearing up my thinking on this point. In particular he suggested that this problem is acute for single case propensity theorists since they, unlike long run propensity theorists, can point to no observable correlate of their theoretical postulates. The long run propensity theorist can provide some empirical motivation for their postulation, even though they choose an empirical correlate (frequencies) with unfortunate features for an analysis of probability. It remains true, as a reviewer points out, that all propensity theorists are inclined toward pure postulation at times, even if the single case propensity theorists are the most inclined.
} 
pervene is a property to be very suspicious of. ${ }^{68}$ For our purposes, though, it is the enormity of the set of bearers of the propensity which is the worry. For this set will include many objects that could have participated in events to confound the outcome, but did not. The toss could have been averted had the coin been crushed, but it was not. Should the device that could have crushed the coin count as a bearer of the propensity since the outcome counterfactually depends on it? Arguably not; but there seems no natural way of ruling them out, since their contribution or lack thereof changes the possibilities and hence the probabilities of various outcomes. Hence the claim that there are too many entities to which a propensity applies.

It is even more suspicious when we consider that sometimes we ascribe chances of coming into existence, say when we consider whether fluctuations in the ground state of quantum field theory will happen to coordinate in such a way as to produce a particle. This has a well-defined probability; but it has no bearer, since the natural entity that has a chance ascribed to it doesn't yet exist, and may never. ${ }^{69}$

\subsection{Against Mellor's Distribution Display Analysis}

Mellor's views avoid many of the problems we have launched against other single case views, simply in virtue of his insistence that propensities cannot be postulated alone and in isolation from other properties of the experimental setup. Rather, propensities supervene on the arrangement of other properties of the system, like the frequency of outcomes or the physical symmetries, allowing a Humean basis for propensity assignments. His analysis also avoids the claim that it merely stipulates propensities (at least insofar as any theoretical term can avoid being postulated in some sense), since the substantive properties that propensities endow their bearers with are actually grounded in the supervenience base of the propensity. In virtue of this, one might begin to suspect that 'propensity' in Mellor's account is a very different thing than in the accounts of Popper, Giere \&c., and that for Mellor it merely names some complex theoretical term, definable from already understood terms (Lewis, 1970). Nevertheless, worries remain with several features of the account.

19. Distributions Displayed? Mellor's account requires that dispositions manifest each time they are trialed, so he requires that the probability distribution appear on each run of the experiment. This must mean that the physical property upon

\footnotetext{
${ }^{68}$ Chris Hitchcock suggested this worry.

${ }^{69}$ Karen Bennett suggested this general worry.
} 
which the distribution function supervenes is completely displayed. But this leaves unanswered the question as to how this physical property uniquely underlies this distribution, if the single case propensity is to be counterfactually independent of other instances. And if the distribution is somehow metaphysically fixed in some other way then Mellor owes us an answer as to what feature determines the distribution. I understand what it means for a frequency distribution to be partially displayed in each trial: each trial completely manifests the underlying feature that supports the frequency assignment. I don't understand what else might be happening in Mellor's case than this, and I don't understand how his account can avoid the same problems. ${ }^{70}$

20. Subjectivity. In his criticism of Mellor, Salmon (1979) makes the point that the detour through subjective probability to ground objective chance is problematic. For in the absence of an accepted probability distribution, the only constraint on rational subjective credence is coherence with the axioms of probability. Whether this is empirically reasonable can only be found out after some constraint is placed on the subjective credences; and the only resource we have while constructing the theory is the frequency evidence, and perhaps the symmetries of the situation. ${ }^{71}$ But the use of symmetry principles in constraining credences is at least problematic, as reflection on the history of the principle of indifference indicates (van Fraassen, 1989: ch. 12). The brief lesson here is that symmetries can only be unproblematically used if we already possess a theoretical model of the situation which allows certain symmetry transformations, and such a model itself needs to be confirmed by frequency evidence. As for the frequencies, it is possible for the frequency to arbitrarily diverge from the probability introduced in the theory and still be evidence for it; there is no logical link between the evidence and the reasonable credence. Furthermore, in the absence of an analysis of probability, it is not even possible to quantify the possibility of the divergence of frequency from probability so as to reassure us that the problem is never very bad (e.g. by using the law of large numbers). For all we can know on the basis of coherent credence, the only evidence we have in probabilistic theory construction might be arbitrarily far from the actual value of probability we try to ascertain. Intuitively, then, the credences we have can be arbitrarily far from the genuine propensity values; as such, Mellor's approach seems not to provide a reliable empirical constraint that would make the postulation of theoretical propensities legitimate.

\footnotetext{
${ }^{70}$ This argument is dual to argument 12. Thanks to Alan Hájek for discussion.

${ }^{71}$ As a reviewer points out.
} 
Mellor does have a response to this: his infamous principle of connectivity (Mellor, 1971: 114-50). The principle is at best obscurely stated: it seems to mean that propensities will be connected with a set of other properties that will be necessary and sufficient for the presence of the propensity. In an updated terminology, this amounts at least to the claim that propensities supervene on this set of properties. In the case of a coin's bias, which is a propensity, these properties could include an uneven mass distribution, some physical warping by heat, or an irregular magnetic field surrounding the tossing device. This connection imposes

a regulative principle that any deviation from equality, however slight, in the chances of heads and tails is to be explained by asymmetry in other properties.

(Mellor, 1971: 127)

This regulative principle is not based on ignorance or indifference, according to Mellor, and should not be susceptible to paradoxes such as those discussed by van Fraassen. If so, then the connections that Mellor's principle relies on cannot be a priori, but must be part of a theoretical model of the situation that is already possessed. That the labelling of a coin is irrelevant to its bias, but its crooked shape is relevant, is a substantive assumption about the causal relations amongst those properties. This model needs itself some empirical confirmation; the fact that the properties of crookedness and labelling are empirically accessible does not entail that a causal model involving them is so accessible. Even if the relation of relevance is not causal, it must have some modal dimension, including, at the least, probabilistic relevance between the events of possessing each property. And the difficulty of giving a non-frequency justification of such a model is exactly Salmon's point that we began with. Connectivity, though it may be a true claim about the metaphysics of probabilistic properties and their supervenience bases, does not help with the methodological issue that Salmon is pointing to. This is especially so since symmetries and connected properties are at best defeasible evidence for probabilities-frequencies can defeat symmetries, but arguably not vice versa (Lewis, 1994: 229).

21. Subjectivity Again. The detour through rational credences makes for another problem. Recently a number of authors have criticised the adequacy of the standard probability calculus for credences: some have wished to move from perfectly precise probabilities to interval-valued or 'vague' probabilities (van Fraassen, 1990; Walley, 1991); some have wanted to reject countable additivity for credences (de Finetti, 1974), and have thus rejected conglomerability (Schervish et al., 1984). 
If the arguments of these authors are accepted, then credences obey a related but different probability calculus. If we need to go via credence to get chance, the chance will also inherit these features. But chance does not have these features, and it would not play the role that it actually does in science if it were to have these features. Moreover, features like vagueness are standardly thought of as linguistic or epistemic phenomena. What ontic feature could, if known, legitimise a subjectively vague probability distribution; yet such credences can nevertheless be rational in the face of the evidence.

\section{Conclusion}

What I hope to have shown is that despite their promise, propensity analyses face a number of difficulties. These difficulties derive from details about each particular implementation, as well as from very general features that all the implementations share. The diversity of arguments provided against these analyses indicates that there are problems with propensity as an analysis of probability for a broad range of philosophical positions concerning empiricism, laws and chances: so broad a range that I doubt that all the premises of the foregoing arguments can be coherently maintained at one time. But my aim is not to defend the individual premises, but to maintain the conclusion that propensity interpretations are untenable.

Of course, some of the arguments are more compelling than others, which itself indicates a fall back position for the defender of propensities as an interpretation of probability. This position will be dictated by exactly which arguments one finds convincing, and hence the premises of which one will deny. On this front, it seems to me that the best chances for a viable propensity interpretation will involve repudiating empiricist demands for a straightforward non-metaphysical interpretation of the disposition display and of the truth-makers for probability propositions. This may result in a propensity interpretation that construes propensities as primitive de re probabilistic causal powers of relational arrays of individuals. I think that if this is the best hope for a propensity interpretation of probability, then that is enough for a reductio. But even if it is not a reductio, it does place quite strong constraints on what type of propensity interpretation can be maintained. It should at least be dismaying how much philosophical baggage one has to accept in order to analyse probability in terms of propensities. ${ }^{72}$

\footnotetext{
${ }^{72}$ Thanks to audiences at Princeton and the Adelaide AAP. Particular thanks go to Alan Hájek, Bas van Fraassen, Jeff Speaks, Chris Hitchcock, Daniel Nolan, David Chalmers, Adam Elga, Karen
} 


\section{References}

Albert, David Z. (2000), Time and Chance. Cambridge, MA: Harvard University Press.

Armstrong, D. M. (1997), A World of States of Affairs. Cambridge: Cambridge University Press.

Arnauld, Antoine and Nicole, Pierre (1996), Logic or the Art of Thinking. Cambridge Texts in the History of Philosophy, Cambridge: Cambridge University Press. Translated and edited by Jill Vance Buroker.

Carnap, Rudolf (1962), Logical Foundations of Probability. Chicago: University of Chicago Press, 2nd ed.

Clark, Peter (2001), "Statistical Mechanics and the Propensity Interpretation of Probability". In Jean Bricmont et al. (eds.), Chance in Physics: Foundations and Perspectives, Berlin: Springer, pp. 271-81.

de Finetti, Bruno (1964), "Foresight: Its Logical Laws, Its Subjective Sources". In Henry E. Kyburg, Jr. and Howard E. Smokler (eds.), Studies in Subjective Probability, New York: Wiley, pp. 93-158.

(1974), Theory of Probability. New York: Wiley.

Dickson, W. Michael (1998), Quantum Chance and Non-Locality. Cambridge: Cambridge University Press.

Eagle, Antony (2004), "Twenty-One Arguments Against Propensity Analyses of Probability”. Erkenntnis, vol. 60: pp. 371-416.

Earman, John (1986), A Primer on Determinism. Dordrecht: D. Reidel.

Eells, Ellery (1983), "Objective Probability Theory Theory". Synthese, vol. 57: pp. 387-442.

Fetzer, James H. (1971), “Dispositional Probabilities”. In R. Buck and R. Cohen (eds.), PSA 1970, Dordrecht: D. Reidel, pp. 473-82. (1981), Scientific Knowledge. Dordrecht: D. Reidel.

Field, Hartry (2003), "Causation in a Physical World". In Michael J. Loux and Dean Zimmerman (eds.), Oxford Handbook of Metaphysics, Oxford: Oxford University Press, pp. 435-60.

Bennett, Richard Corry, Mark Johnston, Lizzie Maughan, Gill Russell, Mark Schroeder, Zena Hitz, Vera Koffman and two anonymous reviewers. I also wish to thank the Boyce Gibson Memorial Library. 
Giere, Ronald N. (1973), "Objective Single-Case Probabilities and the Foundations of Statistics". In Patrick Suppes et al. (eds.), Logic, Methodology and Philosophy of Science, vol. 4, Amsterdam: North-Holland, pp. 467-83.

_ (1976), "A Laplacean Formal Semantics for Single Case Propensities". Journal of Philosophical Logic, vol. 5: pp. 321-53.

Gillies, Donald (2000), Philosophical Theories of Probability. London: Routledge.

Hacking, Ian (1965), The Logic of Statistical Inference. Cambridge: Cambridge University Press.

(1975), The Emergence of Probability. Cambridge: Cambridge University Press.

Hájek, Alan (1997), “'Mises Redux'-Redux: Fifteen Arguments Against Finite Frequentism”. Erkenntnis, vol. 45: pp. 209-227.

- (2003a), "Conditional Probability Is the Very Guide of Life". In Henry E. Kyburg, Jr. and Mariam Thalos (eds.), Probability is the Very Guide of Life, Chicago: Open Court, pp. 183-203.

- (2003b), "What Conditional Probability Could Not Be". Synthese, vol. 137: pp. 273-323.

_ (forthcoming), "The Reference Class Problem is Your Problem Too". Synthese.

(unpublished), "Fifteen Arguments Against Hypothetical Frequentism". Ms., Division of Humanities and Social Sciences, CalTech.

Howson, Colin (1984), "Probabilities, Propensities and Chance". Erkenntnis, vol. 21: pp. 279-94.

Howson, Colin and Urbach, Peter (1993), Scientific Reasoning: the Bayesian Approach. Chicago: Open Court, 2 ed.

Hughes, R. I. G. (1989), The Structure and Interpretation of Quantum Mechanics. Cambridge, MA: Harvard University Press.

Humphreys, Paul W. (1985), "Why Propensities Cannot be Probabilities". Philosophical Review, vol. 94: pp. 557-70.

Ismael, Jenann (1996), "What Chances Could Not Be". British Journal for the Philosophy of Science, vol. 47: pp. 79-91.

Jackson, Frank and Pargetter, Robert (1982), "Physical Probability as a Propensity”. Noûs, vol. 16: pp. 567-83. 
Jeffrey, Richard C. (1977), "Mises Redux". In Robert E. Butts and Jaakko Hintikka (eds.), Basic Problems in Methodology and Linguistics, Dordrecht: D. Reidel, pp. 213-222.

(2004), Subjective Probability (The Real Thing). Cambridge: Cambridge University Press.

King, Jeffrey C. (1998), "What is a Philosophical Analysis?” Philosophical Studies, vol. 90: pp. 155-79.

Kolmogorov, A. N. (1956), Foundations of the Theory of Probability. New York: Chelsea, 2 ed.

Kyburg, Jr., Henry E. (1974), "Propensities and Probabilities". British Journal for the Philosophy of Science, vol. 25: pp. 358-75.

Levi, Isaac (1980), The Enterprise of Knowledge. Cambridge, MA: MIT Press.

__ (1990), "Chance". Philosophical Topics, vol. 18: pp. 117-49.

Lewis, David (1970), "How to Define Theoretical Terms". Journal of Philosophy, vol. 67: pp. 427-46.

(1973), “Causation”. In Lewis (1986), pp. 159-213.

- (1980), "A Subjectivist's Guide to Objective Chance”. In Lewis (1986), pp. 83-132.

_ (1986), Philosophical Papers, vol. 2. Oxford: Oxford University Press.

_ (1994), “Humean Supervenience Debugged”. In Lewis (1999), pp. 224-46.

— (1997), "Finkish Dispositions”. In Lewis (1999), pp. 133-51.

(1999), Papers in Metaphysics and Epistemology. Cambridge: Cambridge University Press.

Loewer, Barry (2004), "David Lewis' Humean Theory of Objective Chance". Philosophy of Science, vol. 71: pp. 1115-25.

Martin, C. B. (1994), "Dispositions and Conditionals". Philosophical Studies, vol. 44: pp. 1-8.

(1997), "On the Need for Properties: The road to Pythagoreanism and back". Synthese, vol. 112: pp. 193-231.

McCurdy, C. S. I. (1996), "Humphreys' Paradox and the Interpretation of Inverse Conditional Probability". Synthese, vol. 108: pp. 105-25.

Mellor, D. H. (1971), The Matter of Chance. Cambridge: Cambridge University Press. 
(1995), The Facts of Causation. New York: Routledge.

Miller, D. W. (1996), “Propensities and Indeterminism”. In A. O' Hear (ed.), Karl Popper: Philosophy and Problems, Cambridge: Cambridge University Press, pp. 121-47.

Milne, P. (1985), "Can there be a Realist Single Case Interpretation of Probability?" Erkenntnis, vol. 25: pp. 129-32.

Mumford, Stephen (1998), Dispositions. Oxford: Oxford University Press.

Peirce, Charles Sanders (1910), "Notes on the Doctrine of Chances". In Justus Buchler (ed.), Philosophical Writings of Peirce, New York: Dover, pp. 164-73.

Pollock, John L. (1990), Nomic Probability and the Foundations of Induction. New York: Oxford University Press.

Popper, Karl (1959a), The Logic of Scientific Discovery. London: Hutchinson. (1959b), "A Propensity Interpretation of Probability". British Journal for the Philosophy of Science, vol. 10: pp. 25-42.

_ (1990), A World of Propensities. Bristol: Thoemmes.

Prior, Elizabeth W., Pargetter, Robert and Jackson, Frank (1982), "Three Theses About Dispositions". American Philosophical Quarterly, vol. 19: pp. 251-7.

Ramsey, F. P. (1990), “Truth and Probability". In Philosophical Papers, Cambridge, UK: Cambridge University Press, pp. 52-94.

Reichenbach, Hans (1949), The Theory of Probability. Berkeley: University of California Press.

Salmon, Wesley C. (1979), "Propensities: a Discussion Review of Mellor (1971)". Erkenntnis, vol. 14: pp. 183-216.

Schervish, M. J., Seidenfeld, T. and Kadane, J. B. (1984), "The Extent of NonConglomerability of Finitely Additive Probability". Zeitschrift für Wahrscheinlichkeitstheorie und Verwandte Gebiete, vol. 66: pp. 205-26.

Shoemaker, Sydney (1980), "Causality and Properties". In Peter van Inwagen (ed.), Time and Cause, Dordrecht: D. Reidel, pp. 109-32.

Sklar, Lawrence (1993), Physics and Chance. Cambridge: Cambridge University Press.

Strevens, Michael (1998), "Inferring Probabilities From Symmetries". Noûs, vol. 32: pp. 231-46. 
Tooley, Michael (1987), Causation: a Realist Approach. Oxford: Oxford University Press.

van Fraassen, Bas C. (1980), The Scientific Image. Oxford: Oxford University Press.

(1989), Laws and Symmetry. Oxford: Oxford University Press.

(1990), "Figures in a Probability Landscape". In J. Michael Dunn and Anil Gupta (eds.), Truth or Consequences, Dordrecht: Kluwer, pp. 345-56.

(1991), Quantum Mechanics: An Empiricist View. Oxford: Oxford University Press.

von Mises, Richard (1957), Probability, Statistics and Truth. New York: Dover.

Walley, P. (1991), Statistical Reasoning with Imprecise Probabilities. London: Chapman and Hall.

Weiner, Matthew and Belnap, Nuel (2006), "How Causal Probabilities Might Fit into Our Objectively Indeterministic World”. Synthese, vol. 149: pp. 1-36. 